\title{
THIN FILM EPITAXY WITH OR WITHOUT SLOPE SELECTION
}

\author{
BO LI AND JIAN-GUO LIU
}

\begin{abstract}
Two nonlinear diffusion equations for thin film epitaxy, with or without slope selection, are studied in this work. The nonlinearity models the Ehrlich-Schwoebel effect- the kinetic asymmetry in attachment and detachment of adatoms to and from terrace boundaries. Both perturbation analysis and numerical simulation are presented to show that such an atomistic effect is the origin of a nonlinear morphological instability, in a roughsmooth-rough pattern, that has been experimentally observed as transient in an early stage of epitaxial growth on rough surfaces. Initial-boundary-value problems for both equations are proven to be well-posed, and the solution regularity is also obtained. Galerkin spectral approximations are studied to provide both a priori bounds for proving the well-posedness and numerical schemes for simulation. Numerical results are presented to confirm part of the analysis and to explore the difference between the two models on coarsening dynamics.
\end{abstract}

\section{INTRODUCTION}

We consider the following two nonlinear diffusion equations - growth equations:

$$
\partial_{t} h=-\nabla \cdot\left(\frac{\nabla h}{1+|\nabla h|^{2}}+\delta \nabla \Delta h\right)
$$

and

$$
\partial_{t} h=-\nabla \cdot\left[\left(1-|\nabla h|^{2}\right) \nabla h+\delta \nabla \Delta h\right]
$$

that model epitaxial growth of thin films, where $h=h(x, t), x=\left(x_{1}, x_{2}\right)$, is a scaled height function of thin film in a co-moving frame and $\delta$ is a positive constant. If the surface gradient $|\nabla h|$ is small, then $1 /(1+|\nabla h|)^{2} \approx 1-|\nabla h|^{2}$, and (1.2) can be formally derived from (1.1). With suitable boundary conditions, solutions of these equations satisfy

$$
\frac{d}{d t} \int h d x=0
$$

i.e., the mass is conserved.

In Eqs. (1.1) and (1.2), the fourth-order term models surface diffusion, and the nonlinear second-order term models the Ehrlich-Schowoebel effect. In a typical step-flow or layer-bylayer epitaxial growth of thin films, adatoms - atoms that are adsorbed onto the surface but have not yet become part of the crystal - diffuse on a terrace and likely hit a terrace boundary. In order to stick to the boundary from an upper terrace, an adatom must overcome a higher energy barrier - the Ehrlich-Schwoebel barrier [7,30,31], cf. Figure 1.1. This asymmetry in attachment and detachment of adatoms to and from terrace boundaries has many important consequences: it induces an uphill current which in general destabilizes nominal surfaces (high symmetry surfaces) $[7,30,31]$, but stabilizes vicinal surfaces (surfaces that are in the vicinity

Date: April 22, 2003. 


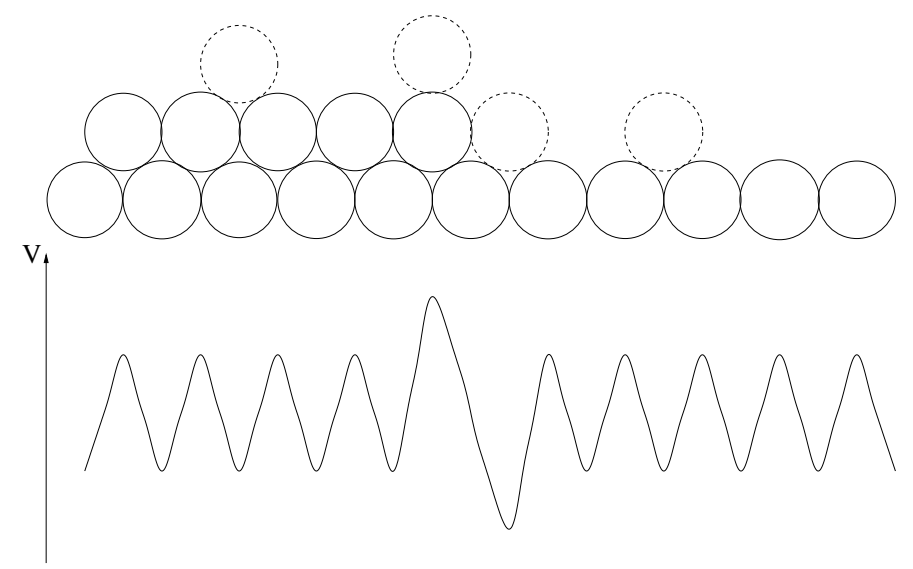

Figure 1.1. The Ehrlich-Schwoebel barrier.

of high symmetry surfaces) with large slope, preventing step bunching [35]; it also leads to the Bales-Zangwill morphological instability of atomic steps [2,29]; Finally, it contributes to the kinetic roughening of film surfaces $[17,27,35]$.

The growth equations (1.1) and (1.2) are the gradient flow with respect to the $L^{2}(\Omega)$ inner product of energy functionals

$$
E_{1}(h)=\int_{\Omega}\left[-\frac{1}{2} \ln \left(1+|\nabla h|^{2}\right)+\frac{\delta}{2}|\Delta h|^{2}\right] d x
$$

and

$$
E_{2}(h)=\int_{\Omega}\left[\frac{1}{4}\left(|\nabla h|^{2}-1\right)^{2}+\frac{\delta}{2}|\Delta h|^{2}\right] d x,
$$

respectively. Due to the presence of the negative term, the energy $E_{1}(h)$ is expected to behave badly, and is in fact poorly understood mathematically. The second energy $E_{2}(h)$ appears in several areas of material modeling. For instance, it serves as a variational model in the theory of liquid crystals [1]. It is an example of elastic energy functional of scalar deformations $h$ in the strain-gradient theory for structural phase transitions in solids $[3,19]$. It is also a simplified and rescaled folding energy for an out-of-plane displacement $h$ modelling the folding pattern of a blister formed in the buckling-driven delamination of thin films $[9,14]$. In the context of thin film epitaxy, the first term in $E_{2}(h)$ selects the slope of the film surface. For this reason, we call the associated equation (1.2) the growth equation with slope selection, and correspondingly the equation (1.1) the growth equation without slope selection.

The interfacial dynamics governed by Eqs. (1.1) and by (1.2) are different. With slope selection, Eq. (1.2) predicts that mound-like or pyramid structures in the surface profile tend to have a uniform, constant mound slope $[23,25]$. However, without slope selection, Eq. (1.1) predicts an unbounded mound slope $M(t)=O\left(t^{1 / 4}\right)$ [10]. Experiments and simulations predict that the roughness - the standard deviation of the height profile $w(t)$ and the characteristic lateral size $\lambda(t)$ of pyramids obey the scaling laws $w(t) \sim t^{\beta}$ and $\lambda(t) \sim t^{n}$ before the finite size effect kicks in. These exponents $\beta$ and $n$ are called the roughness exponent and the coarsening exponent, respectively. With slope selection, Eq. (1.2) predicts $\beta=n=1 / 3$; without slope selection, Eq. (1.1) predicts $\beta=1 / 2$ and $n=1 / 4[10,15,21,23,25,32]$. We refer to the recent work [20] for a discussion on mathematical justification of such predictions. 
Both the growth equation (1.2) for thin film epitaxy and the Cahn-Hilliard equation [5] for phase separation in a binary alloy model mass-conserved systems, and have variational structures though with different topologies. For one-dimensional space, the derivative of the solution to the growth equation (1.2) solves the Cahn-Hilliard equation. However, for two-dimensional space, the Cahn-Hilliard equation is not suitable for modeling the epitaxial growth of thin films, cf. the argument in Section 4.6.2 of [28]. The geometry in the epitaxial dynamics (1.2) seems to be more complicated than that in the Cahn-Hilliard dynamics.

In this work, we attempt to understand continuum consequences of the Ehrlich-Schwoebel effect and differences between the two models with regards to solution properties and coarsening dynamics. Our main contributions are as follows.

(1) We show by a high-order perturbation analysis and numerical simulation for both equations that the Ehrlich-Schwoebel effect is the origin of a nonlinear morphological instability, in a rough-smooth-rough pattern, that has been experimentally observed in epitaxial growth of thin film on rough surfaces [11]. This is a typical transient phenomenon in an early stage of thin film epitaxy.

(2) We prove that the initial-boundary-value problem for both of the growth equations is well-posed. We also obtain the solution regularity.

(3) We prove various kinds of bounds and error estimates for Galerkin spectral approximations of the weak solution for both equations. These bounds and estimates are used to prove the well-posedness and justify our numerical computation.

(4) We develop a spectral method for solving numerically the growth equations, and present numerical results that confirm part of our analysis and show differences of the two models in terms of solution behavior and coarsening dynamics.

The well-posedness for the growth equation with slope selection for different boundary conditions is also studied in [18] using a different approach.

We now recall briefly the derivation of the growth equation (1.1) under the following assumptions: (1) a thin film is growing due to an incoming atomic or molecular beam with a constant deposition flux rate $F$; $(2)$ the main mechanism of mass transport of the film is the geometrically controlled, isotropic surface diffusion; and (3) the Ehrlich-Schwoebel effect is significant. All other effects such as the beam fluctuation, adatom desorption, diffusion anisotropy, and island nucleation are neglected. For a growing high-symmetry surface in molecular-beam epitaxy with relatively low temperature and large average size of adatom islands, these assumptions are reasonable [29].

In a fixed Cartesian coordinate system $O x_{1} x_{2} x_{3}$, we represent the macroscopic height of the film surface at time $t$ in a co-moving frame by a height function $x_{3}=h\left(x_{1}, x_{2}, t\right)$. Thus, $h=h_{\text {real }}-F t$, where $h_{\text {real }}$ is the real height of the film surface at time $t$. Conservation of mass leads to

$$
\partial_{t} h=-\nabla \cdot \mathbf{j}
$$

where $\mathbf{j}=\mathbf{j}(\nabla h)$ is the surface current depending on the macroscopic gradient $\nabla h$ of the film surface. Following our assumptions, we have

$$
\mathbf{j}=\mathbf{j}_{S D}+\mathbf{j}_{E S},
$$

where $\mathbf{j}_{S D}$ is the equilibrium surface current due to the adatom surface diffusion and $\mathbf{j}_{E S}$ the kinetic surface current due to the Ehrlich-Schwoebel effect. 
It follows from Fick's law $[16,26]$ that $\mathbf{j}_{S D}=-K_{s} \nabla_{s} \mu_{c}$, where $K_{s}>0$ is a constant, $\nabla_{s}$ denotes the surface gradient, and $\mu_{c}$ is the equilibrium surface chemical potential. Following Herring [12,13] and Mullins [24], we have $\mu_{c}=-\gamma H$, where $\gamma>0$ is the constant surface tension and $H$ the mean curvature of film surface. Linearizing both the surface gradient $\nabla_{s}$ and the mean curvature $H$, we obtain

$$
\mathbf{j}_{S D}=K_{s} \gamma \nabla \Delta h
$$

To determine $\mathbf{j}_{E S}$, we recall the argument in $[15,32]$. For simplicity, consider the step-flow growth of a thin film with an average step width $l$. Let $m=|\nabla h|$ be the macroscopic surface slope and take the lattice parameter to be the unity. Then, $m=1 / l$. Denote also by $\sigma$ the adatom diffusion distance. Assume temporarily an infinite Ehrlich-Schwoebel barrier. For small $m$ for which $\sigma<l$, only the atoms that land on a terrace within distance $\sigma$ of the step edge shared by the adjacent upper terrace will make it to the edge. The rest will attach to islands on the terrace, and will not contribute to the net current. In this case, the total current should be given by the number of atoms which land in strips of width $\sigma$ near an advancing step edge shared by the adjacent upper terrace, $F \sigma$, multiplied by the number of such strips per unit length, $\sigma / l$. Since $m=|\nabla h|=1 / l$, we have that $\mathbf{j}_{E S}=F \sigma^{2} \nabla h$ for small $m$. For large $m$ for which $\sigma>l$, assume that the current has the form $\mathbf{j}_{E S}=F \sigma^{2} f(\sigma m) \nabla h$ for some function $f$. Since in this case all atoms land on terraces will stick to step edges, the current should not depend on the diffusion distance $\sigma$. Thus, $f(s) \sim 1 / s^{2}$ for large values of $s$. To the leading order, this scaling is found to agree with experiments [15]. Consequently, $\mathbf{j}_{E S} \sim\left(F / m^{2}\right) \nabla h$ for large $m$. Assume now a finite Ehrlich-Schwoebel barrier $B_{c}$. As proposed in [15], the corresponding surface current is an interpolation of the two values for small and large $m$, i.e.,

$$
\mathbf{j}_{E S}=\frac{F B_{c} \sigma^{2}}{1+\alpha \sigma^{2}|\nabla h|^{2}} \nabla h
$$

where $\alpha$ is a positive constant independent of $\sigma$. Finally, we obtain (1.1) from (1.5)-(1.8) with suitable scaling.

We remark that the energy functional $E_{2}(h)$ and the related equation (1.2) are also derived in [25] based on a series expansion of flux in powers of the profile gradient, together with the symmetry principle and the enforcement of Onsager's reciprocity relations from statistical mechanics.

In Section 2, we show a nonlinear morphological instability in epitaxial growth on rough surfaces. In Section 3, we prove the well-posedness and solution regularity for the initialboundary-value problem. In Section 4, we prove various kinds of bounds and error estimates for Galerkin spectral approximations. In Section 5, we present numerical results. Finally, in Section 6, we draw conclusions.

\section{Nonlinear Morphological Instability}

An interesting experiment on unstable growth of thin films on rough surfaces is reported in [11]. In this experiment, the molecular-beam epitaxy of InAs buffer layers is performed on $\operatorname{InAs}(001)$ substrates that initially exhibit small wavelength oscillations. Such oscillations remain when the thickness of buffer layers reaches $600 \AA$. They almost disappear when the thickness reaches $1500 \AA$. Much later, when the thickness reaches $2 \mu \mathrm{m}$, new, large wavelength 
oscillations appear. Kinetic Monte Carlo simulations strongly suggest that this morphological instability in the rough-smooth-rough pattern is due to the Ehrlich-Schwoebel effect [11].

Following [6], we now show by a high-order perturbation analysis that such a morphological instability is due to nonlinear interaction of different modes in perturbation, and is captured by the Ehrlich-Schwoebel effect included in both of the growth equations (1.1) and (1.2).

Perturb a constant height function and set

$$
h(x, t)=h_{0}+\epsilon h_{1}(x, t)+\epsilon^{2} h_{2}(x, t)+\epsilon^{3} h_{3}(x, t)+\cdots
$$

with the initial condition

$$
h(x, 0)=h_{0}+\epsilon \sum_{j=1}^{N} a_{j} \exp \left\{i\left(k_{j} \cdot x+\phi_{j}\right)\right\},
$$

where $h_{0}$ is a complex constant, $\epsilon$ is a parameter small in magnitude, $h_{1}, h_{2}$, and $h_{3}$ are complex-valued functions of $x$ and $t, N \geq 2$ is an integer, all $a_{j}(1 \leq j \leq N)$ are complex constants, $\phi_{j}(1 \leq j \leq N)$ are real constants, and $k_{j}(1 \leq j \leq N)$ are real, two-dimensional, constant vectors - the wave-vectors.

Inserting the expression (2.1) into the equation (1.1) or (1.2) and comparing orders of $\epsilon$, also using (2.2), we obtain the following equations and initial conditions for $h_{1}, h_{2}$, and $h_{3}$, the same for both equations (1.1) and (1.2),

$$
\begin{aligned}
\epsilon: & \partial_{t} h_{1}=-\Delta h_{1}-\delta \Delta^{2} h_{1}, \\
& h_{1}(x, 0)=\sum_{j=1}^{N} a_{j} \exp \left\{i\left(k_{j} \cdot x+\phi_{j}\right)\right\}, \\
\epsilon^{2}: \quad & \partial_{t} h_{2}=-\Delta h_{2}-\delta \Delta^{2} h_{2}, \\
& h_{2}(x, 0)=0, \\
\epsilon^{3}: \quad & \partial_{t} h_{3}=\nabla \cdot\left(\left|\nabla h_{1}\right|^{2} \nabla h_{1}\right)-\Delta h_{3}-\delta \Delta^{2} h_{3}, \\
& h_{3}(x, 0)=0 .
\end{aligned}
$$

The $h_{1}$ equation (2.3) is the linearized equation around the steady-state solution $h(x, t)=$ $h_{0}$. Solving the initial-value problem (2.3) and (2.4), we obtain that

$$
h_{1}(x, t)=\sum_{j=1}^{N} a_{j} \exp \left\{\omega\left(k_{j}\right) t+i\left(k_{j} \cdot x+\phi_{j}\right)\right\},
$$

where $\omega(k)$ is the growth rate for wave-vector $k$, determined by the dispersion relation

$$
\omega(k)=|k|^{2}-\delta|k|^{4} .
$$

See Figure 2.1 for a plot of a typical such dispersion relation. There exists a critical value, $k_{c}$, of the modulus of wave-vector,

$$
k_{c}=\sqrt{\frac{1}{\delta}} \quad \text { such that } \begin{array}{ll}
\omega(k)>0 & \text { if }|k|<k_{c} \\
\omega(k)<0 & \text { if }|k|>k_{c} .
\end{array}
$$

The maximum growth rate $\omega_{m}=1 /(4 \delta)$ occurs at any wave-vector with modulus $\sqrt{1 /(2 \delta)}$. 


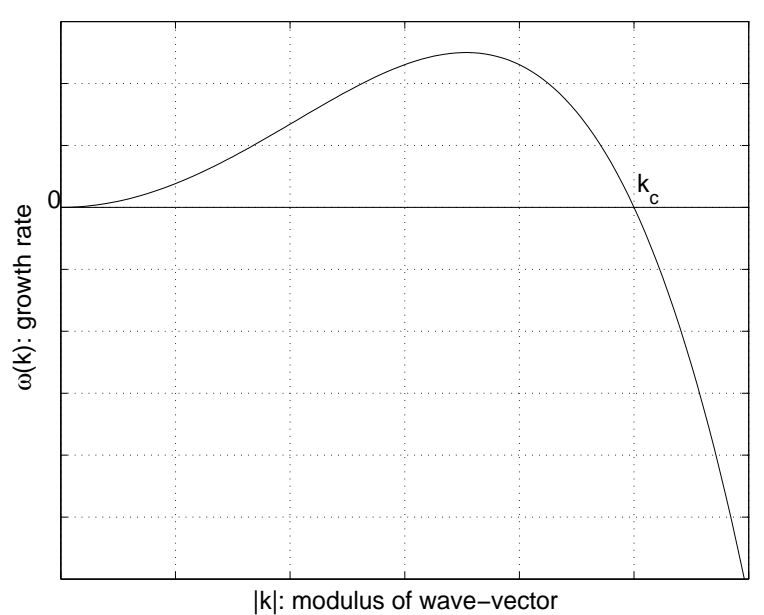

Figure 2.1. A typical dispersion relation.

From (2.5) and (2.6), we immediately have that $h_{2}(x, t)=0$ identically.

The $h_{3}$ equation (2.7) is still linear. But it has a nonlinear source term $\nabla \cdot\left(\left|\nabla h_{1}\right|^{2} \nabla h_{1}\right)$ which comes from the Ehrlich-Schwoebel effect. By (2.9), we have

$$
\begin{aligned}
g(x, t) \equiv & \nabla \cdot\left[\left|\nabla h_{1}(x, t)\right|^{2} \nabla h_{1}(x, t)\right] \\
= & -\sum_{l, m, n=1}^{N} a_{l} a_{m} a_{n}\left(k_{l} \cdot k_{m}\right)\left[k_{n} \cdot\left(k_{l}-k_{m}+k_{n}\right)\right] \\
& \times \exp \left\{\left[\omega\left(k_{l}\right)+\omega\left(k_{m}\right)+\omega\left(k_{n}\right)\right] t+i\left[\left(k_{l}-k_{m}+k_{n}\right) \cdot x+\phi_{l}-\phi_{m}+\phi_{n}\right]\right\} .
\end{aligned}
$$

Applying the Fourier transform to both sides of (2.7) and (2.8), we obtain for each variable $\xi$ of Fourier transformed functions and each time $t$ that

$$
\begin{aligned}
\partial_{t} \widehat{h_{3}}(\xi, t)-\omega(\xi) \widehat{h_{3}}(\xi, t) & =\hat{g}(\xi, t), \\
\widehat{h_{3}}(\xi, 0) & =0,
\end{aligned}
$$

where

$$
\hat{u}(\xi)=\mathcal{F}(u)(\xi)=\frac{1}{\sqrt{2 \pi}} \int_{\mathbb{R}^{2}} u(x) e^{-i \xi \cdot x} d x
$$

is the Fourier transform of a function $u=u(x)$. Solving this initial-value problem for each $\xi$, we get

$$
\widehat{h_{3}}(\xi, t)=\int_{0}^{t} e^{\omega(\xi)(t-\tau)} \hat{g}(\xi, \tau) d \tau .
$$

Consequently, by the inverse Fourier transform, defined for a function $U=U(\xi)$ by

$$
\check{U}(\xi)=\mathcal{F}^{-1}(U)(\xi)=\frac{1}{\sqrt{2 \pi}} \int_{\mathbb{R}^{2}} U(\xi) e^{i x \cdot \xi} d \xi,
$$

we obtain

$$
h_{3}(x, t)=\frac{1}{\sqrt{2 \pi}} \int_{\mathbb{R}^{2}}\left[\int_{0}^{t} e^{\omega(\xi)(t-\tau)} \hat{g}(\xi, \tau) d \tau\right] e^{i x \cdot \xi} d \xi=\int_{0}^{t} \mathcal{F}^{-1}\left(e^{\omega(\xi)(t-\tau)} \hat{g}(\xi, \tau)\right) d \tau .
$$


After a series of calculations, we obtain an explicit expression of $h_{3}(x, t)$, which, together with $(2.1),(2.9)$, and the fact that $h_{2}(x, t)=0$, in turn yields

$$
\begin{aligned}
h(x, t)=h_{0} & +\epsilon \sum_{j=1}^{N} a_{j} \exp \left\{\omega\left(k_{j}\right) t+i\left(k_{j} \cdot x+\phi_{j}\right)\right\} \\
& -\epsilon^{3} \sum_{l, m, n=1}^{N} \frac{a_{l} a_{m} a_{n}\left(k_{l} \cdot k_{m}\right)\left[k_{n} \cdot\left(k_{l}-k_{m}+k_{n}\right)\right]}{\omega\left(k_{l}\right)+\omega\left(k_{m}\right)+\omega\left(k_{n}\right)-\omega\left(k_{l}-k_{m}+k_{n}\right)} \\
& \cdot\left(\exp \left\{\left[\omega\left(k_{l}\right)+\omega\left(k_{m}\right)+\omega\left(k_{n}\right)\right] t\right\}-\exp \left\{\omega\left(k_{l}-k_{m}+k_{n}\right) t\right\}\right) \\
& \cdot \exp \left\{i\left[\left(k_{l}-k_{m}+k_{n}\right) \cdot x+\phi_{l}-\phi_{m}+\phi_{n}\right]\right\}+O\left(\epsilon^{4}\right),
\end{aligned}
$$

provided that

$$
\omega\left(k_{l}\right)+\omega\left(k_{m}\right)+\omega\left(k_{n}\right)-\omega\left(k_{l}-k_{m}+k_{n}\right) \neq 0 \quad \forall l, m, n: 1 \leq l, m, n \leq N .
$$

Suppose that all the wave-vectors $k_{j}(1 \leq j \leq N)$ in the initial condition (2.2) satisfy (2.12) and

$$
\left|k_{j}\right|>k_{c} \quad \forall j: 1 \leq j \leq N
$$

Then, all $\omega\left(k_{j}\right)<0(1 \leq j \leq N)$ by (2.10). Consequently, all the $\epsilon$ terms in (2.11) decay exponentially. However, a careful look at the expression (2.11) finds that in fact all the $\epsilon^{3}$ terms in (2.11) also decay exponentially except those with indices $l, m$, and $n$ satisfying

$$
0<\left|k_{l}-k_{m}+k_{n}\right|<k_{c}
$$

If this holds for some indices $l, m$, and $n$, then a new, unstable mode with the wavenumber $k_{l}-k_{m}+k_{n}$ will be generated. Since $\epsilon^{3}$ is much smaller than $\epsilon$, it takes a relatively long period of "buffering" time - during which the surface appears to be smooth - for the new oscillation to be observed.

This nonlinear morphological instability, characterized by the rough-smooth-rough growth pattern, models accurately the experimentally observed transient in early stage of thin film epitaxy on rough surface as described above. It is clear from our analysis that this instability is due to the nonlinear interaction of different modes in the perturbation and that the nonlinearity originates from the Ehrlich-Schwoebel effect. If an initial perturbation contains modes with both large and small wavenumbers, then the small wavenumber component of the perturbation will grow quickly and the new, unstable modes generated by the nonlinear interaction of those modes with large wavenumbers will also grow exponentially but at a much later time. Therefore, both kinds of unstable modes, old and new, will develop but in different temporal scales.

Figure 2.2 shows a sequence of snapshots of the surface height at different time from our numerical simulation of the initial-boundary-value problem of the one-dimensional growth equation with slope selection

$$
\begin{array}{ll}
h_{t}=\left(\left(h_{x}\right)^{3}-h_{x}-h_{x x x}\right)_{x} & \forall(x, t) \in(0,12) \times(0, T], \\
h(\cdot, t) \text { is 12-periodic } & \forall t \in[0, T], \\
h(x, 0)=0.1\left(\sin \frac{\pi x}{2}+\sin \frac{2 \pi x}{3}+\sin \pi x\right) & \forall x \in[0,12],
\end{array}
$$



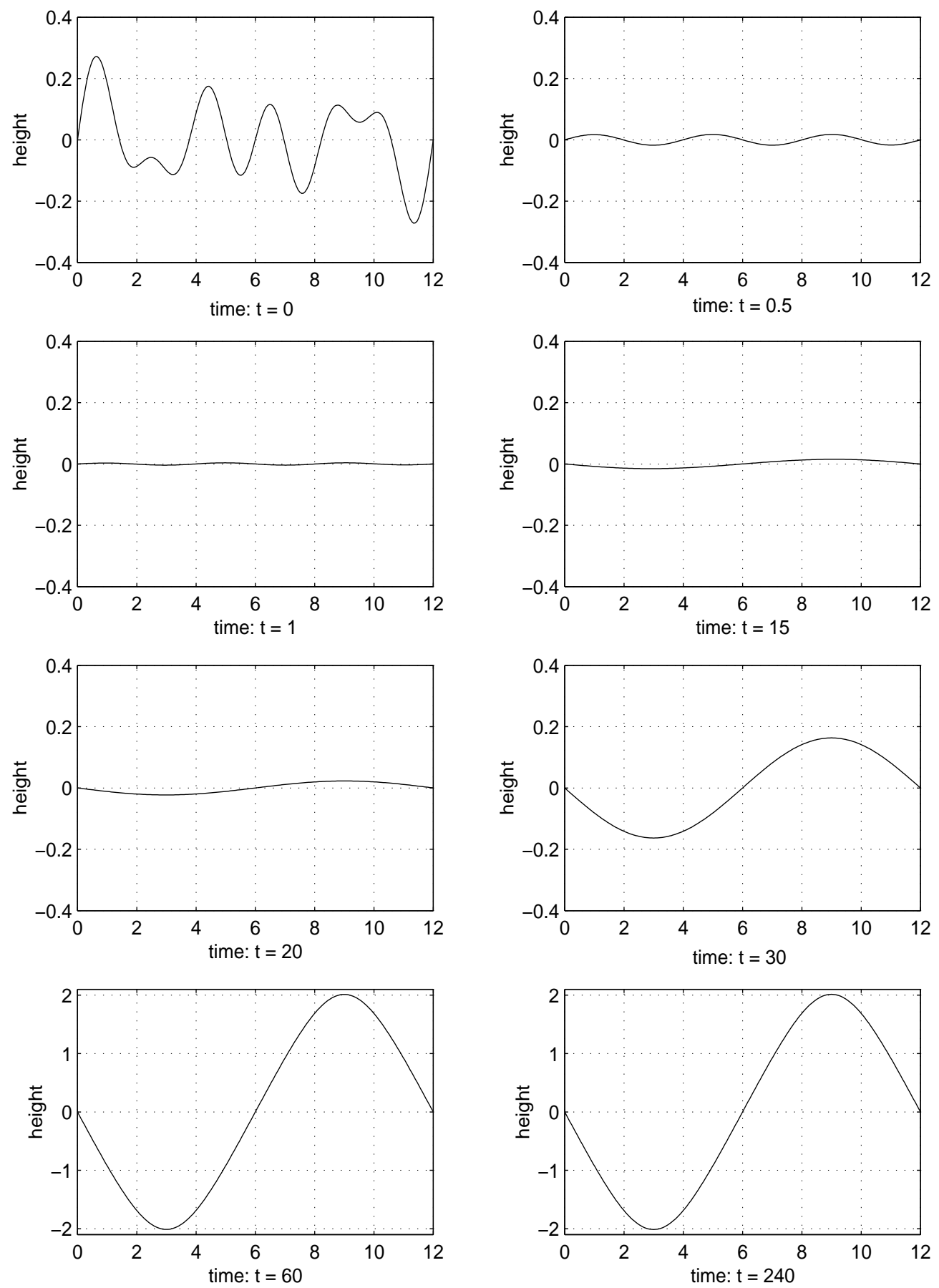

Figure 2.2. A morphological instability due to nonlinear interaction.

where a subscript $t$ or $x$ denotes a corresponding partial derivative and $T>0$ is a constant. In all the plots, the horizontal axis is the $x$ axis. We see that the initial oscillation is damped immediately at $t=1$. After a relatively long period of "buffering" time, from $t=1$ to $t=15$, a new kind of oscillation appears, and then exponentially increases. Notice that all 
the wavenumbers $k_{1}, k_{2}$, and $k_{3}$ in the initial condition used in the simulation satisfy (2.12) and (2.13) for $N=3$ with the critical wavenumber $k_{c}=1$. Moreover, only one ordered index triple $(l, m, n)$ with $1 \leq l, m, n \leq N$ satisfies (2.14). This is why the new oscillation consists of one sinusoid profile. In the last two plots for $t=60$ and $t=240$, the height different from that of the others. It is clear that a steady-state is reached at around $t=60$.

Define the roughness (or the interface width, cf. [4]) for the height function at time $t$ by

$$
w(t)=\sqrt{\frac{1}{L} \int_{0}^{L}[h(x, t)-\bar{h}(t)]^{2} d x}, \quad \text { where } \bar{h}(t)=\frac{1}{L} \int_{0}^{L} h(x, t) d x
$$

with $L=12$. The two plots in Figure 2.3 show in different scales the evolution of film surface roughness, in particular, the rough-smooth-rough pattern. The flat part at long time in the right plot shows that a steady-state solution is reached.
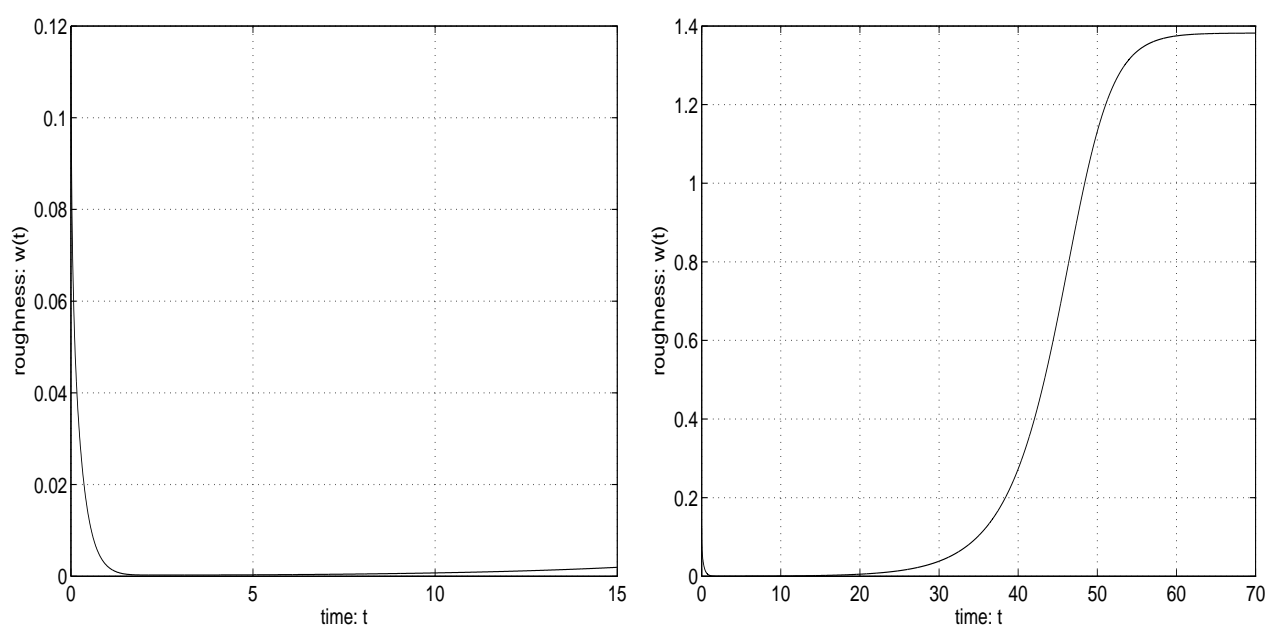

Figure 2.3. The evolution of the surface roughness.

\section{The Initial-Boundary-Value Problem}

Consider the initial-boundary-value problem for the $d$-dimensional "growth" equation

$$
\begin{aligned}
& \partial_{t} h=-\nabla \cdot\left[\left(1-G\left(|\nabla h|^{2}\right)\right) \nabla h+\delta \nabla \Delta h\right] \quad \text { in } \Omega \times(0, T], \\
& h(\cdot, t) \text { is } L \text {-periodic for all } t \in[0, T], \\
& h(x, 0)=h_{0}(x) \quad \forall x \in \Omega,
\end{aligned}
$$

where $h: \bar{\Omega} \times[0, T] \rightarrow \mathbb{R}$ is a $d$-dimensional "height" function in a co-moving frame, $\Omega=$ $\prod_{j=1}^{d}\left(0, L_{j}\right) \subset \mathbb{R}^{d}, d=1,2$, or $3, \mathbb{R}$ denotes the set of all real numbers, $L=\left(L_{1}, \cdots, L_{d}\right)$, all $L_{1}, \cdots, L_{d}, \delta$, and $T$ are positive constants, $h_{0}: \Omega \rightarrow \mathbb{R}$ is a given function, and

$$
G(s)= \begin{cases}G_{1}(s)=\frac{s}{1+s} & \text { without slope selection, } \\ G_{2}(s)=s & \text { with slope selection. }\end{cases}
$$

Here, we rearrange the second-order term in Eq. (1.1), and use $G(\cdot)$ to unify notation. 
Let $C_{\text {per }}^{\infty}(\bar{\Omega})$ be the set of all restrictions onto $\bar{\Omega}$ of all real-valued, $L$-periodic, $C^{\infty}$-functions on $\mathbb{R}^{d}$. For any integer $m \geq 0$ and any extended real number $p \in[1, \infty]$, let $W_{\text {per }}^{m, p}(\Omega)$ be the closure of $C_{\text {per }}^{\infty}(\bar{\Omega})$ in the usual Sobolev space $W^{m, p}(\Omega)$. Note that $W_{\text {per }}^{0, p}(\Omega)=L^{p}(\Omega)$, if $p \neq \infty$. For $m \geq 1$ and $p=2$, we write $H_{p e r}^{m}(\Omega)$ instead of $W_{p e r}^{m, 2}(\Omega)$, and denote by $H_{p e r}^{-m}(\Omega)$ the dual space of $H_{p e r}^{m}(\Omega)$. Throughout the paper, we denote by $\|\cdot\|$ the $L^{2}(\Omega)$-norm. We also write a function $u: \Omega \times[0, T] \rightarrow \mathbb{R}$ which is in a function space $X$ for each $t$ as a mapping $u=u(t):[0, T] \rightarrow X$.

Definition 3.1 (Weak solution). A function $h: \Omega \times[0, T] \rightarrow \mathbb{R}$ is called a weak solution of the initial-boundary-value problem (3.1)-(3.3), if

(1) $h \in L^{2}\left(0, T ; H_{p e r}^{2}(\Omega)\right)$ and $\partial_{t} h \in L^{2}\left(0, T ; H_{p e r}^{-2}(\Omega)\right)$;

(2) for any $\phi \in H_{\text {per }}^{2}(\Omega)$,

$$
\left\langle\phi, \partial_{t} h\right\rangle+\left\langle\nabla \phi,\left(G\left(|\nabla h|^{2}\right)-1\right) \nabla h\right\rangle+\delta\langle\Delta \phi, \Delta h\rangle=0 \quad \text { a.e. } t \in(0, T),
$$

where, without confusion, $\langle\cdot, \cdot\rangle$ denotes the action of a distribution on a test function or the inner product of $L^{2}(\Omega)$;

(3) $h(x, 0)=h_{0}(x)$ for a.e. $x \in \Omega$.

Theorem 3.1 (Existence, uniqueness, and energy identity). Let $h_{0} \in H_{p e r}^{2}(\Omega)$. Then, the initial-boundary-value problem (3.1)-(3.3) has a unique weak solution $h: \Omega \times[0, T] \rightarrow \mathbb{R}$ that satisfies the following energy identities:

$$
\begin{aligned}
& \frac{d}{d t}\|h\|^{2}+4 E_{1}(h)+2 \int_{\Omega}\left[\frac{1}{1+|\nabla h|^{2}}+\ln \left(1+|\nabla h|^{2}\right)\right] d x=2|\Omega| \quad \text { a.e. } t \in(0, T), \\
& \frac{d}{d t} E_{1}(h)+\left\|\partial_{t} h\right\|^{2}=0 \quad \text { a.e. } t \in(0, T),
\end{aligned}
$$

and

$$
\begin{aligned}
& \frac{d}{d t}\|h\|^{2}+4 E_{2}(h)+\|\nabla h\|_{L^{4}(\Omega)}^{4}=|\Omega| \quad \text { a.e. } t \in(0, T), \\
& \frac{d}{d t} E_{2}(h)+\left\|\partial_{t} h\right\|^{2}=0 \quad \text { a.e. } t \in(0, T),
\end{aligned}
$$

for Eqs. (1.1) and (1.2), respectively, where $E_{1}(h), E_{2}(h)$ are given in $(1.3),(1.4)$, and $|\Omega|$ is the measure of $\Omega$.

Theorem 3.2 (Stability). Let $g_{0}, h_{0} \in H_{\text {per }}^{2}(\Omega)$. Let $g, h$ be the weak solutions of Eqs. (3.1) and $(3.2)$ with $g(\cdot, 0)=g_{0}$ and $h(\cdot, 0)=h_{0}$ a.e. $\Omega$, respectively. Then,

$$
\|g-h\|_{L^{\infty}\left(0, T ; L^{2}(\Omega)\right)}+\|g-h\|_{L^{2}\left(0, T ; H^{2}(\Omega)\right)} \leq C\left\|g_{0}-h_{0}\right\|_{L^{2}(\Omega)} .
$$

Theorem 3.3 (Regularity). Let $h_{0} \in H_{\text {per }}^{m}(\Omega)$ for some integer $m \geq 2$. Then, the weak solution $h: \Omega \times[0, T] \rightarrow \mathbb{R}$ of the initial-boundary-value problem (3.1)-(3.3) satisfies

$$
h \in L^{\infty}\left(0, T ; H^{m}(\Omega)\right) \cap L^{2}\left(0, T ; H^{m+2}(\Omega)\right) \quad \text { and } \partial_{t} h \in L^{2}\left(0, T ; H^{m-2}(\Omega)\right) .
$$

For $d \geq 4$, we still have the existence, uniqueness, energy identities, and stability for the two equations. But for Eq. (1.2) (with slope selection), both the definition of a weak solution and the assumption on initial data must be modified: in addition, we need to assume in the definition of a weak solution that $\nabla h \in L^{4}\left(0, T ; L^{4}(\Omega)\right)$ and that the test 
function $\phi$ is such that $\nabla \phi \in L^{4}\left(0, T ; L^{4}(\Omega)\right)$. We need also to assume that the initial data $h_{0} \in H_{p e r}^{r(d)}(\Omega)$, where $r(d)$ is the smallest integer that greater than or equal to $1+d / 4$. Note that $H^{r(d)}(\Omega) \hookrightarrow W^{1,4}(\Omega)$.

Slightly changing the definition of a weak solution by requiring that $\partial_{t} h \in L^{1}\left(0, T ; H_{\text {per }}^{-2}(\Omega)\right)$ instead of $\partial_{t} h \in L^{2}\left(0, T ; H_{p e r}^{-2}(\Omega)\right)$, we can prove the existence of a weak solution with a much weaker assumption: $h_{0} \in L^{2}(\Omega)$. The proof will need a refined compactness result that is obtained in, e.g., [33].

To prove the above and other theorems, we need some preparation. First, we recall the following elementary result.

Lemma 3.1. We have for any $\phi \in H_{\text {per }}^{2}(\Omega)$ that

$$
\begin{aligned}
& \|\nabla \phi\|^{2} \leq\|\phi\|\|\Delta \phi\| \leq \frac{1}{2}\|\phi\|^{2}+\frac{1}{2}\|\Delta \phi\|^{2}, \\
& \sum_{j, k=1}^{d}\left\|\partial_{x_{j} x_{k}} \phi\right\|^{2}=\|\Delta \phi\|^{2} .
\end{aligned}
$$

Now denote for each integer $N \geq 1$

$$
H_{N}=\operatorname{span}\{1, \cos (2 \pi k \cdot x / L), \sin (2 \pi k \cdot x / L): 0<|k| \leq N\},
$$

where $k=\left(k_{1}, \cdots, k_{d}\right) \in \mathbb{Z}^{d}$ with $k_{j} \geq 0(1 \leq j \leq d), \mathbb{Z}$ is the set of all integers, $x / L$ denotes the vector $\left(x_{1} / L_{1}, \cdots, x_{d} / L_{d}\right)$, and $|k|=\sum_{j=1}^{d} k_{j}$. Notice that $H_{N} \subset C_{p e r}^{\infty}(\bar{\Omega})$. Denote also by $\mathcal{P}_{N}: L^{2}(\Omega) \rightarrow H_{N}$ the $L^{2}(\Omega)$-projection onto $H_{N}$, which is defined for any $u \in L^{2}(\Omega)$ by $\mathcal{P}_{N} u \in H_{N}$ and

$$
\left\langle\mathcal{P}_{N} u-u, \phi\right\rangle=0 \quad \forall \phi \in H_{N} .
$$

Lemma 3.2. We have for any integer $m \geq 0$ that

$$
\begin{aligned}
& \left\|\mathcal{P}_{N} u\right\|_{H^{m}(\Omega)} \leq\|u\|_{H^{m}(\Omega)} \quad \forall u \in H_{\text {per }}^{m}(\Omega), \forall N \geq 1, \\
& \lim _{N \rightarrow \infty}\left\|\mathcal{P}_{N} u-u\right\|_{H^{m}(\Omega)}=0 \quad \forall u \in H_{p e r}^{m}(\Omega) .
\end{aligned}
$$

Proof. The case $m=0$ is trivial. For $m \geq 1$, these results follow from the fact that any derivative of a function in $H_{N}$ is still in $H_{N}$ and that $\mathcal{P}_{N} \partial=\partial \mathcal{P}_{N}$ for any derivative $\partial$.

The following lemma treats the nonlinearity in Eqs. (1.1) and (1.2). It can be proved by direct verification.

Lemma 3.3. We have for any vectors $a, b \in \mathbb{R}^{d}$ that

$$
\begin{aligned}
& 2(a-b) \cdot\left(\frac{|a|^{2} a}{1+|a|^{2}}-\frac{|b|^{2} b}{1+|b|^{2}}\right) \\
& \quad=\frac{\left(|a|^{2}-|b|^{2}\right)^{2}}{\left(1+|a|^{2}\right)\left(1+|b|^{2}\right)}+|a-b|^{2}\left(\frac{|a|^{2}}{1+|a|^{2}}+\frac{|b|^{2}}{1+|b|^{2}}\right), \\
& (a-b) \cdot\left(|a|^{2} a-|b|^{2} b\right)=|a|^{2}|b|^{2}-(a \cdot b)^{2} \\
& \quad+((a-b) \cdot a)^{2}+((a-b) \cdot b)^{2}+((a-b) \cdot a)((a-b) \cdot b),
\end{aligned}
$$


and, in particular, that

$$
(a-b) \cdot(G(a) a-G(b) b) \geq 0 .
$$

Given any integer $k \geq 1$, we denote $\left|\nabla^{k} u\right|=\sum_{|\gamma|=k}\left|\partial^{\gamma} u\right|$ for any smooth function $u$. For each integer $j$ with $1 \leq j \leq k$, we also denote by $\Lambda_{k, j}$ the set of all pairs $(\alpha, \beta)$ with $\alpha=\left\{\alpha_{i}\right\}$ and $\beta=\left\{\beta_{i}\right\}$ two finite sequences of integers such that $1 \leq \alpha_{i} \leq k+1-j$ for all $i \geq 1$ and $\sum_{i \geq 1} \alpha_{i} \beta_{i}=k$, and denote $\Lambda_{k}=\cup_{j=1}^{k} \Lambda_{k, j}$.

Lemma 3.4. Given any integer $k \geq 1$, there exists a constant $C_{k}>0$ such that

$$
\left|\nabla^{k} G_{2}(u(x))\right| \leq C_{k} \sum_{\left(\left\{\alpha_{i}\right\},\left\{\beta_{i}\right\}\right) \in \Lambda_{k}} \prod_{i \geq 1}\left|\nabla^{\alpha_{i}} u(x)\right|^{\beta_{i}} \quad \forall x \in \Omega, \forall u \in C^{k}(\Omega) .
$$

Proof. Fix $u \in C^{k}(\bar{\Omega})$ and $x \in \Omega$. Denote by $\partial^{k}$ any partial derivative of order $k$. We have by induction that

$$
\partial^{k}\left[G_{2}(u(x))\right]=\sum_{j=1}^{k} \sum_{\left(\left\{\alpha_{i}\right\},\left\{\beta_{i}\right\}\right) \in \Lambda_{k, j}} C_{k, j,\left\{\alpha_{i}\right\},\left\{\beta_{i}\right\}} G^{(j)}(u(x)) \prod_{i \geq 1}\left(\nabla^{\alpha_{i}} u(x)\right)^{\beta_{i}},
$$

where $C_{k, j,\left\{\alpha_{i}\right\},\left\{\beta_{i}\right\}}$ are all constants independent of $u$. This, together with the fact that $\left|G_{2}^{(j)}(s)\right| \leq 1$ for any integer $j \geq 1$ and any $s \geq 0$, implies the desired inequality.

We now proceed to prove Theorems 3.1-3.3. Our proofs rely heavily on the a priori bounds on spectral approximations of weak solutions. Such bounds will be fully established in the next section. Throughout the paper, we denote by $C$ a generic, positive constant that can in general depend on $d, L_{j}(1 \leq j \leq d), T, \delta$, and $h_{0}$, but not on $N$.

Proof of Theorem 3.1. It follows from Theorem 4.1 that there exist a sequence of functions $\left\{h_{N}\right\}_{N=1}^{\infty} \subset L^{\infty}\left(0, T ; H_{p e r}^{2}(\Omega)\right)$ with $\left\{\partial_{t} h_{N}\right\} \subset L^{2}\left(0, T ; L^{2}(\Omega)\right)$ such that for each $N \geq 1$ $h_{N}(\cdot, 0)=h_{0} ;$ for any $\psi_{N} \in H_{N}$

$$
\left\langle\psi_{N}, \partial_{t} h_{N}\right\rangle+\left\langle\nabla \psi_{N},\left[G\left(\left|\nabla h_{N}\right|^{2}\right)-1\right] \nabla h_{N}\right\rangle+\delta\left\langle\Delta \psi_{N}, \Delta h_{N}\right\rangle=0 \quad \text { a.e. } t \in(0, T) ;
$$

and

$$
\left\|h_{N}\right\|_{L^{\infty}\left(0, T ; H^{2}(\Omega)\right)}+\left\|\partial_{t} h_{N}\right\|_{L^{2}\left(0, T ; L^{2}(\Omega)\right)} \leq C .
$$

Consequently, there exists $h \in L^{\infty}\left(0, T ; H_{\text {per }}^{2}(\Omega)\right)$ with $\partial_{t} h \in L^{2}\left(0, T ; L^{2}(\Omega)\right)$ such that

$$
\begin{array}{ll}
h_{N} \stackrel{*}{\rightarrow} h & \text { in } L^{\infty}\left(0, T ; H^{2}(\Omega)\right), \\
\partial_{t} h_{N} \rightarrow \partial_{t} h & \text { in } L^{2}\left(0, T ; L^{2}(\Omega)\right), \\
h_{N} \rightarrow h & \text { in } L^{2}\left(0, T ; H^{1}(\Omega)\right),
\end{array}
$$

where the strong convergence (3.9) follows from (3.8), the weak convergence $h_{N} \rightarrow h$ in $L^{2}\left(0, T ; H^{2}(\Omega)\right)$ which follows from (3.7), and a usual compactness result (cf. [34], Theorem 2.1, Chapter III). So, Part (1) of Definition 3.1 is satisfied.

Let $\phi \in H_{\text {per }}^{2}(\Omega)$ and $\eta \in C[0, T]$. For each $N \geq 1$, set $\psi_{N}=\mathcal{P}_{N} \phi$ in (3.5), multiply both sides of the resulting identity by $\eta(t)$, and integrate against $t$ to yield

$$
\int_{0}^{T}\left\langle\eta(t) \mathcal{P}_{N} \phi, \partial_{t} h_{N}(\cdot, t)\right\rangle d t+\int_{0}^{T}\left\langle\eta(t) \nabla \mathcal{P}_{N} \phi, G\left(\left|\nabla h_{N}(\cdot, t)\right|^{2}\right) \nabla h_{N}(\cdot, t)\right\rangle d t
$$




$$
-\int_{0}^{T}\left\langle\eta(t) \nabla \mathcal{P}_{N} \phi, \nabla h_{N}(\cdot, t)\right\rangle d t+\delta \int_{0}^{T}\left\langle\eta(t) \Delta \mathcal{P}_{N} \phi, \Delta h_{N}(\cdot, t)\right\rangle d t=0 .
$$

It directly follows from Lemma 3.2, (3.7), and (3.9) that

$$
\begin{aligned}
& \int_{0}^{T}\left\langle\eta(t) \mathcal{P}_{N} \phi, \partial_{t} h_{N}(\cdot, t)\right\rangle d t \rightarrow \int_{0}^{T}\left\langle\eta(t) \phi, \partial_{t} h(\cdot, t)\right\rangle d t \quad \text { as } N \rightarrow \infty, \\
& \int_{0}^{T}\left\langle\eta(t) \nabla \mathcal{P}_{N} \phi, \nabla h_{N}(\cdot, t)\right\rangle d t \rightarrow \int_{0}^{T}\langle\eta(t) \nabla \phi, \nabla h(\cdot, t)\rangle d t \quad \text { as } N \rightarrow \infty, \\
& \int_{0}^{T}\left\langle\eta(t) \Delta \mathcal{P}_{N} \phi, \Delta h_{N}(\cdot, t)\right\rangle d t \rightarrow \int_{0}^{T}\langle\eta(t) \Delta \phi, \Delta h(\cdot, t)\rangle d t \quad \text { as } N \rightarrow \infty .
\end{aligned}
$$

By the Hölder inequality, the Sobolev embedding $H^{2}(\Omega) \hookrightarrow W^{1,4}(\Omega)$, Lemma 3.2, (3.6)-(3.9), and the fact that $G(s) \leq s$ for all $s \geq 0$, we deduce that

$$
\begin{aligned}
& \left|\int_{0}^{T}\left\langle\eta(t) \nabla \mathcal{P}_{N} \phi, G\left(\left|\nabla h_{N}(\cdot, t)\right|^{2}\right) \nabla h_{N}(\cdot, t)\right\rangle d t-\int_{0}^{T}\left\langle\eta(t) \nabla \phi, G\left(|\nabla h(\cdot, t)|^{2}\right) \nabla h(\cdot, t)\right\rangle d t\right| \\
& \leq\left|\int_{0}^{T}\left\langle\eta(t)\left[\nabla \mathcal{P}_{N} \phi-\nabla \phi\right], G\left(\left|\nabla h_{N}(\cdot, t)\right|^{2}\right) \nabla h_{N}(\cdot, t)\right\rangle d t\right| \\
& \quad+\left|\int_{0}^{T}\left\langle\eta(t) \nabla \phi, G\left(\left|\nabla h_{N}(\cdot, t)\right|^{2}\right)\left[\nabla h_{N}(\cdot, t)-\nabla h(\cdot, t)\right]\right\rangle d t\right| \\
& \quad+\left|\int_{0}^{T}\left\langle\eta(t) \nabla \phi,\left[G\left(\left|\nabla h_{N}(\cdot, t)\right|^{2}\right)-G\left(|\nabla h(\cdot, t)|^{2}\right)\right] \nabla h(\cdot, t)\right\rangle d t\right| \\
& \quad \leq\|\eta\|_{L^{\infty}(0, T)}\left\|\mathcal{P}_{N} \phi-\phi\right\|_{W^{1,4}(\Omega)} \int_{0}^{T}\left\|\nabla h_{N}\right\|_{L^{4}(\Omega)}^{3} d t \\
& \quad+\|\eta\|_{L^{\infty}(0, T)}\|\phi\|_{W^{1, \infty}(\Omega)}\left\|\nabla h_{N}\right\|_{L^{4}\left(0, T ; L^{4}(\Omega)\right)}^{2}\left\|\nabla h_{N}-\nabla h\right\|_{L^{2}\left(0, T ; L^{2}(\Omega)\right)} \\
& \quad+\|\eta\|_{L^{\infty}(0, T)}\|\phi\|_{W^{1, \infty}(\Omega)}\left(\left\|\nabla h_{N}\right\|_{L^{4}\left(0, T ; L^{4}(\Omega)\right)}^{2}+\|\nabla h\|_{L^{4}\left(0, T ; L^{4}(\Omega)\right)}^{2}\right)\left\|h_{N}-h\right\|_{L^{2}\left(0, T ; H^{1}(\Omega)\right)} \\
& \leq \\
& \quad C\left\|\mathcal{P}_{N} \phi-\phi\right\|_{H^{2}(\Omega)}+C\left\|h_{N}-h\right\|_{L^{2}\left(0, T ; H^{1}(\Omega)\right)} \\
& \quad 0 \quad \text { as } N \rightarrow \infty .
\end{aligned}
$$

Letting $N \rightarrow \infty$ in (3.10), we get from (3.11)-(3.14) that

$$
\begin{aligned}
& \int_{0}^{T} \eta(t)\left\{\left\langle\phi, \partial_{t} h(\cdot, t)\right\rangle+\left\langle\nabla \phi,\left[G\left(|\nabla h(\cdot, t)|^{2}\right)-1\right] \nabla h(\cdot, t)\right\rangle\right. \\
& \quad+\delta\langle\Delta \phi, \Delta h(\cdot, t)\rangle\} d t=0 .
\end{aligned}
$$

Since $\eta \in C[0, T]$ is arbitrary, this implies (3.4). Part (2) of Definition 3.1 is satisfied.

Notice that, after a possible modification of $h$ on a set of measure zero, we have $h \in$ $C\left([0, T] ; L^{2}(\Omega)\right)$. Moreover, $h(t)=h(s)+\int_{s}^{t} h^{\prime}(\tau) d \tau$ for any $s, t \in[0, T]$, where $h(t)=$ $h(\cdot, t) \in L^{2}(\Omega)$ and $h^{\prime}(t)=\partial_{t} h(\cdot, t)$, (cf. [8], Theorem 2, Section 5.9). Replace $\eta(t)$ in $(3.15)$ by $\eta_{T}(t)=-t / T+1$ and integrate by parts against $t$ for the first term to get

$$
\int_{0}^{T} \frac{1}{T}\langle\phi, h(\cdot, t)\rangle d t+\int_{0}^{T} \eta_{T}(t)\left\{\left\langle\nabla \phi,\left[G\left(|\nabla h(\cdot, t)|^{2}\right)-1\right] \nabla h(\cdot, t)\right\rangle+\delta\langle\Delta \phi, \Delta h(\cdot, t)\rangle\right\} d t
$$




$$
=\langle\phi, h(\cdot, 0)\rangle \text {. }
$$

Repeat the same argument using (3.5) with $\psi_{N}=\mathcal{P}_{N} \phi$ to deduce with $\phi$ replaced by $\mathcal{P}_{N} \phi$ and use Part (3) of Theorem 4.1 to deduce

$$
\begin{aligned}
\int_{0}^{T} & \frac{1}{T}\left\langle\mathcal{P}_{N} \phi, h_{N}(\cdot, t)\right\rangle d t+\int_{0}^{T} \eta_{T}(t)\left\{\left\langle\nabla \mathcal{P}_{N} \phi,\left[G\left(\left|\nabla h_{N}(\cdot, t)\right|^{2}-1\right] \nabla h_{N}(\cdot, t)\right\rangle\right.\right. \\
& \left.+\delta\left\langle\Delta \mathcal{P}_{N} \phi, \Delta h_{N}(\cdot, t)\right\rangle\right\} d t=\left\langle\mathcal{P}_{N} \phi, h_{0}\right\rangle .
\end{aligned}
$$

Thus, taking the limit $N \rightarrow \infty$, we deduce from (3.7), (3.9), and (3.12)-(3.14) that

$$
\begin{aligned}
\int_{0}^{T} & \frac{1}{T}\langle\phi, h(\cdot, t)\rangle d t+\int_{0}^{T} \eta_{T}(t)\left\{\left\langle\nabla \phi,\left[G\left(|\nabla h(\cdot, t)|^{2}\right)-1\right] \nabla h(\cdot, t)\right\rangle+\delta\langle\Delta \phi, \Delta h(\cdot, t)\rangle\right\} d t \\
& =\left\langle\phi, h_{0}\right\rangle .
\end{aligned}
$$

Now, a comparison of (3.16) and (3.17), together with the arbitrariness of $\phi \in H_{p e r}^{2}(\Omega)$, shows that Part (3) in Definition 3.1 is also satisfied. Thus, $h$ is a weak solution.

The uniqueness follows from the stability established in Theorem 3.2. The energy identities can be obtained by setting $\phi=h(\cdot, t)$ and $\phi=\partial_{t} h(\cdot, t)$ for each $t \in(0, T]$ in (3.4), respectively, and using the definition of $E_{1}(h)$ and $E_{2}(h)$.

Proof of Theorem 3.2. Let $w=g-h$. Since $g$ and $h$ are two weak solutions, we have for any $\phi \in H_{\text {per }}^{2}(\Omega)$ and a.e. $t \in(0, T)$ that

$$
\left\langle\phi, \partial_{t} w\right\rangle+\left\langle\nabla \phi,\left(G\left(|\nabla g|^{2}\right) \nabla g-G\left(|\nabla h|^{2}\right) \nabla h\right)\right\rangle-\langle\nabla \phi, \nabla w\rangle+\delta\langle\Delta \phi, \Delta w\rangle=0 .
$$

Since $w \in L^{2}\left(0, T ; H_{p e r}^{2}(\Omega)\right)$ and $\partial_{t} w \in L^{2}\left(0, T ; L^{2}(\Omega)\right), \frac{d}{d t}\langle w, w\rangle=2\left\langle w, \partial_{t} w\right\rangle$ (cf. [8], Theorem 3, Section 5.9). Setting $\phi=w(\cdot, t)$ for each $t \in(0, T]$ and applying Lemma 3.3 with $a=\nabla g$ and $b=\nabla h$, we deduce that

$$
\frac{1}{2} \frac{d}{d t}\|w\|^{2}-\|\nabla w\|^{2}+\delta\|\Delta w\|^{2} \leq 0, \quad \text { a.e. } t \in(0, T) .
$$

But, by integration by parts, the Cauchy-Schwarz inequality, and Young's inequality,

$$
\|\nabla w\|^{2}=-\langle w, \Delta w\rangle \leq\|w\|\|\Delta w\| \leq \frac{1}{2 \delta}\|w\|^{2}+\frac{\delta}{2}\|\Delta w\|^{2} .
$$

This implies that

$$
\frac{d}{d t}\|w\|^{2}-\frac{1}{2 \delta}\|w\|^{2}+\frac{\delta}{2}\|\Delta w\|^{2} \leq 0 \quad \text { a.e. } t \in(0, T) .
$$

The desired inequality now follows from the Gronwall inequality.

Proof of Theorem 3.3. Since the unique weak solution is a weak limit of finite-dimensional approximations whose bounds are established in Theorem 4.1 and Theorem 4.2, the assertion of theorem follows. 


\section{Galerkin Spectral Approximations}

In this section, we define Galerkin spectral approximations, and prove bounds and error estimates for such approximations.

Theorem 4.1 (Galerkin spectral approximations). Let $h_{0} \in H_{\text {per }}^{2}(\Omega)$. For each integer $N \geq 1$, there exists a unique $h_{N}: \Omega \times[0, T] \rightarrow \mathbb{R}$ such that

(1) $h_{N} \in C^{\infty}(\bar{\Omega} \times[0, T])$ and $h_{N}(\cdot, t) \in H_{N}$ for any $t \in[0, T]$;

(2) for any $\phi \in H_{N}$ and any $t \in(0, T]$,

$$
\left\langle\phi, \partial_{t} h_{N}\right\rangle+\left\langle\nabla \phi,\left(G\left(\left|\nabla h_{N}\right|^{2}\right)-1\right) \nabla h_{N}\right\rangle+\delta\left\langle\Delta \phi, \Delta h_{N}\right\rangle=0
$$

(3) $h_{N}(\cdot, 0)=\mathcal{P}_{N} h_{0}$;

$$
\left\|h_{N}\right\|_{L^{\infty}\left(0, T ; H^{2}(\Omega)\right)}+\left\|\partial_{t} h_{N}\right\|_{L^{2}\left(0, T ; L^{2}(\Omega)\right)} \leq C .
$$

We shall call $h_{N}:[0, T] \rightarrow H_{N}$ for each $N \geq 1$ the (semi-discrete) Galerkin spectral approximation of the weak solution $h: \Omega \times[0, T] \rightarrow \mathbb{R}$ of the initial-boundary-value problem (3.1)-(3.2).

Theorem 4.2 (Bounds for Galerkin spectral approximations). Let $h_{0} \in H_{\text {per }}^{m}(\Omega)$ for some integer $m \geq 2$. For each integer $N \geq 1$, let $h_{N}:[0, T] \rightarrow H_{N}$ be the Galerkin spectral approximation of the weak solution $h: \Omega \times[0, T] \rightarrow \mathbb{R}$ of the initial-boundary-value problem (3.1)-(3.2). Then,

$$
\left\|h_{N}\right\|_{L^{\infty}\left(0, T ; H^{m}(\Omega)\right)}+\left\|h_{N}\right\|_{L^{2}\left(0, T ; H^{m+2}(\Omega)\right)}+\left\|\partial_{t} h_{N}\right\|_{L^{2}\left(0, T ; H^{m-2}(\Omega)\right)} \leq C .
$$

Theorem 4.3 (Error estimates). Let $m \geq 4$ be an integer and $h \in L^{\infty}\left(0, T ; H^{m}(\Omega)\right)$ be a weak solution of the initial-boundary-value problem (3.1)-(3.3). For each integer $N \geq 1$, let $h_{N}:[0, T] \rightarrow H_{N}$ be the Galerkin spectral approximation of $h$. Then, we have

$$
\left\|h-h_{N}\right\|_{L^{\infty}\left(0, T ; L^{2}(\Omega)\right)}+\left\|h-h_{N}\right\|_{L^{2}\left(0, T ; H^{2}(\Omega)\right)} \leq C N^{-m}
$$

where the constant $C$ can depend on the solution $h$.

Proof of Theorem 4.1. Let $s(N)=\operatorname{dim}\left(H_{N}\right)$ and $\left\{\phi_{j}\right\}_{j=1}^{s(N)}$ be an orthonormal basis of $H_{N}$ with respect to the $L^{2}(\Omega)$ inner product. Consider

$$
h_{N}(x, t)=\sum_{j=1}^{s(N)} a_{j}(t) \phi_{j}(x)
$$

with some functions $a_{j}=a_{j}(t)(1 \leq j \leq s(N))$ to be determined. Set $\phi=\phi_{j}$ in (4.1) for each $j(1 \leq j \leq s(N))$ and use the orthogonality of $\left\{\phi_{j}\right\}_{j=1}^{s(N)}$ to obtain

$$
a_{j}^{\prime}(t)=f_{j}\left(a_{1}(t), \ldots, a_{s(N)}(t)\right), \quad j=1, \ldots, s(N),
$$

where all $f_{j}: \mathbb{R}^{s(N)} \rightarrow \mathbb{R}(1 \leq j \leq s(N))$ are smooth and locally Lipschitz. Set

$$
a_{j}(0)=\left\langle h_{0}, \phi_{j}\right\rangle, \quad j=1, \ldots, s(N),
$$

which is equivalent to Part (3). It follows from the theory for initial-value problems of ordinary differential equations that there exists $T_{N}>0$ such that the initial-value problem, (4.4) and (4.5), has a unique smooth solution $\left(a_{1}(t), \ldots, a_{s(N)}(t)\right)$ for $t \in\left[0, T_{N}\right]$. 
For each $t \in\left[0, T_{N}\right]$, set $\phi=h_{N}(\cdot, \tau) \in H_{N}$ in (4.1) and use Lemma 3.1 together with Young's inequality to get

$$
\frac{1}{2} \frac{d}{d t}\left\|h_{N}\right\|^{2}+\int_{\Omega}\left|\nabla h_{N}\right|^{2} G\left(\left|\nabla h_{N}\right|^{2}\right) d x+\delta\left\|\Delta h_{N}\right\|^{2}=\left\|\nabla h_{N}\right\|^{2} \leq \frac{C}{2}\left\|h_{N}\right\|^{2}+\frac{\delta}{2}\left\|\Delta h_{N}\right\|^{2} .
$$

Multiply both sides of the inequality by $2 e^{-C t}$ and integrate against $t$ to deduce that

$$
\left\|h_{N}(\cdot, t)\right\|^{2}+\int_{0}^{t} \int_{\Omega}\left|\nabla h_{N}\right|^{2} G\left(\left|\nabla h_{N}\right|^{2}\right) d x+\left\|\Delta h_{N}\right\|_{L^{2}\left(0, T ; L^{2}(\Omega)\right)}^{2} \leq C\left(1+e^{C T}\right)
$$

for all $t \in\left[0, T_{N}\right]$. This, together with the orthogonality of $\left\{\phi_{j}\right\}_{j=1}^{s(N)}$, implies that

$$
\sum_{j=1}^{s(N)}\left[a_{j}(t)\right]^{2}=\left\|h_{N}(\cdot, t)\right\|^{2} \leq C\left(1+e^{C T}\right) \quad \forall t \in\left[0, T_{N}\right] .
$$

The solution $\left(a_{1}(t), \ldots, a_{s(N)}(t)\right)$ of the initial-value problem, (4.4) and (4.5), is thus bounded on $\left[0, T_{N}\right]$, and hence can be uniquely extended to a smooth solution over $[0, \infty)$. Parts (1)(3) are proved.

Replacing $T_{N}$ by $T$ in (4.6) and applying Lemma 3.1, we then obtain that

$$
\left\|h_{N}\right\|_{L^{\infty}\left(0, T ; L^{2}(\Omega)\right)}+\left\|h_{N}\right\|_{L^{2}\left(0, T ; H^{2}(\Omega)\right)} \leq C .
$$

Set now $\phi=\partial_{t} h_{N}(\cdot, t)$ in (4.1) to get for any $t \in[0, T]$ that

$$
\left\|\partial_{t} h_{N}\right\|^{2}+\frac{d}{d t} E(h(\cdot, t))=0,
$$

where $E(h)=E_{1}(h)$ for Eq. (1.1) (without slope selection) and $E(h)=E_{2}(h)$ for Eq. (1.2) (with slope selection). Integrating against $t$, noting that $\ln (1+s) \leq s$ for all $s \geq 0$, and using Lemma 3.1, (4.7), and Young's inequality, we obtain that

$$
\left\|\partial_{t} h\right\|_{L^{2}\left(0, T ; L^{2}(\Omega)\right)}^{2}+\left\|h_{N}\right\|_{L^{\infty}\left(0, T ; H^{2}(\Omega)\right)}^{2} \leq C .
$$

This and (4.7)imply (4.2). Part (4) is proved.

Proof of Theorem 4.2. We proceed by induction on $m \geq 2$. Assume $m=2$. Denote by $\partial$ any first-order spatial partial derivative. Set $\phi=\partial^{2} h_{N}(\cdot, t) \in H_{N}$ in (4.1) for each $t \in(0, T]$ and integrate by parts to get

$$
\frac{1}{2} \frac{d}{d t}\left\|\partial h_{N}\right\|^{2}+\int_{\Omega} \nabla \partial h_{N} \cdot \partial\left[G\left(\left|\nabla h_{N}\right|^{2}\right) \nabla h_{N}\right] d x-\left\|\nabla \partial h_{N}\right\|^{2}+\delta\left\|\Delta \partial h_{N}\right\|^{2}=0 .
$$

Since

$$
\nabla \partial h_{N} \cdot \partial\left[G\left(\left|\nabla h_{N}\right|^{2}\right) \nabla h_{N}\right]=\left|\nabla \partial h_{N}\right|^{2} G\left(\left.\nabla \partial h_{N}\right|^{2}\right)+2\left(\nabla \partial h_{N} \cdot \nabla \partial h_{N}\right)^{2} G^{\prime}\left(\left|\nabla h_{N}\right|^{2}\right) \geq 0,
$$

we obtain by integrating over $t \in[0, T]$ and using Lemma 3.1, Lemma 3.2, and (4.2) that

$$
\left\|h_{N}\right\|_{L^{2}\left(0, T ; H^{3}(\Omega)\right)} \leq C \text {. }
$$

Similarly, using $\phi=\Delta^{2} h_{N}(\cdot, t) \in H_{N}$ for $t \in(0, T)$, we get by Lemma 3.2, (4.2), (4.8), the generalized Hölder inequality, and the Sobolev embedding $H^{1}(\Omega) \hookrightarrow L^{6}(\Omega)$ that

$$
\delta \int_{0}^{T}\left\|\Delta^{2} h_{N}\right\|^{2} d t \leq C+\int_{0}^{T} \int_{\Omega}\left|\Delta^{2} h_{N} \nabla \cdot\left(G\left(\left|\nabla h_{N}\right|^{2}\right) \nabla h_{N}\right)\right| d x d t
$$




$$
\begin{aligned}
& \leq C+C \int_{0}^{T} \int_{\Omega}\left|\Delta^{2} h_{N}\right|\left|\nabla h_{N}\right|^{2}\left|\nabla^{2} h_{N}\right| d x d t \\
& \leq C+C \int_{0}^{T}\left\|\Delta^{2} h_{N}\right\|\left\|h_{N}\right\|_{W^{1,6}(\Omega)}^{2}\left\|h_{N}\right\|_{W^{2,6}(\Omega)} d t \\
& \leq C+C \int_{0}^{T}\left\|\Delta^{2} h_{N}\right\|\left\|h_{N}\right\|_{H^{2}(\Omega)}^{2}\left\|h_{N}\right\|_{H^{3}(\Omega)} d t \\
& \leq C+\frac{\delta}{2} \int_{0}^{T}\left\|\Delta^{2} h_{N}\right\|^{2} d t+C\left\|h_{N}\right\|_{L^{2}\left(0, T ; H^{3}(\Omega)\right)}^{2} .
\end{aligned}
$$

The estimate (4.3) for $m=2$ now follows from Lemma 3.1, (4.9), and (4.2).

We now prove (4.3) for $m \geq 3$ under the assumption that $h_{0} \in H_{\text {per }}^{m}(\Omega)$ and

$$
\left\|h_{N}\right\|_{L^{\infty}\left(0, T ; H^{m-1}(\Omega)\right)}+\left\|h_{N}\right\|_{L^{2}\left(0, T ; H^{m+1}(\Omega)\right)}+\left\|\partial_{t} h_{N}\right\|_{L^{2}\left(0, T ; H^{m-3}(\Omega)\right)} \leq C .
$$

Setting $\phi=\left(\partial^{m}\right)^{2} h_{N}(\cdot, t) \in H_{N}$ in (4.1) for each $t \in(0, T]$, integrating by parts in space, and integrating over $[0, T]$ in time, we have by Lemma 3.1, Lemma 3.2, and (4.10) that

$$
\begin{aligned}
& \left\|h_{N}\right\|_{L^{\infty}\left(0, T ; H^{m}(\Omega)\right)}^{2}+\left\|h_{N}\right\|_{L^{2}\left(0, T ; H^{m+2}(\Omega)\right)}^{2} \\
& \leq C+C \int_{0}^{T} \int_{\Omega}\left|\nabla^{m+2} h_{N}\right|\left|\nabla^{m-1}\left[G\left(\left|\nabla h_{N}\right|^{2}\right) \nabla h_{N}\right]\right| d x d t \\
& \leq C+C \int_{0}^{T} \int_{\Omega}\left|\nabla^{m+2} h_{N}\right|\left|\nabla^{m} h_{N}\right|\left|G\left(\left|\nabla h_{N}\right|^{2}\right)\right| d x d t \\
& \quad+C \sum_{l=1}^{m-1} \int_{0}^{T} \int_{\Omega}\left|\nabla^{m+2} h_{N}\right|\left|\nabla^{m-l} h_{N}\right|\left|\nabla^{l}\left[G\left(\left|\nabla h_{N}\right|^{2}\right)\right]\right| d x d t
\end{aligned}
$$

It follows from the generalized Hölder inequality, the Sobolev embedding $H^{m+1}(\Omega) \hookrightarrow$ $W^{m+1,6}(\Omega)$, and the assumption (4.10) that

$$
\begin{aligned}
& \int_{0}^{T} \int_{\Omega}\left|\nabla^{m+2} h_{N}\right|\left|\nabla^{m} h_{N}\right|\left|G\left(\left|\nabla h_{N}\right|^{2}\right)\right| d x d t \\
& \quad \leq C \int_{0}^{T} \int_{\Omega}\left|\nabla^{m+2} h_{N}\right|\left|\nabla^{m} h_{N}\right|\left|\nabla h_{N}\right|^{2} d x d t \\
& \quad \leq C \int_{0}^{T}\left\|h_{N}\right\|_{H^{m+2}(\Omega)}\left\|h_{N}\right\|_{W^{m, 6}(\Omega)}\left\|h_{N}\right\|_{W^{1,6}(\Omega)}^{2} d t \\
& \quad \leq C \int_{0}^{T}\left\|h_{N}\right\|_{H^{m+2}(\Omega)}\left\|h_{N}\right\|_{H^{m+1}(\Omega)}\left\|h_{N}\right\|_{H^{m}(\Omega)}^{2} d t \\
& \quad \leq C \int_{0}^{T}\left\|h_{N}\right\|_{H^{m+2}(\Omega)}\left\|h_{N}\right\|_{H^{m+1}(\Omega)} d t .
\end{aligned}
$$

This, together with (4.10), (4.11), and Young's inequality, leads to

$$
\left\|h_{N}\right\|_{L^{\infty}\left(0, T ; H^{m}(\Omega)\right)}^{2}+\left\|h_{N}\right\|_{L^{2}\left(0, T ; H^{m+2}(\Omega)\right)}^{2}
$$




$$
\leq C \sum_{l=1}^{m-1} \int_{0}^{T} \int_{\Omega}\left|\nabla^{m+2} h_{N}\right|\left|\nabla^{m-l} h_{N}\right|\left|\nabla^{l}\left[G\left(\left|\nabla h_{N}\right|^{2}\right)\right]\right| d x d t
$$

Assume first that $m=3$. Since $d /(d+3) \in(0,1 / 2)$, we can use the Gagliardo-Nirenberg inequality (cf. e.g., [22], Theorem 1, Section 1.4.8) and the Sobolev embedding to get

$$
\left\|h_{N}\right\|_{W^{2,6}(\Omega)} \leq C\left\|h_{N}\right\|_{W^{3,6}(\Omega)}^{d /(d+3)}\left\|h_{N}\right\|_{H^{2}(\Omega)}^{3 /(d+3)} \leq C\left\|h_{N}\right\|_{H^{4}(\Omega)}^{1 / 2}\left\|h_{N}\right\|_{H^{2}(\Omega)}^{3 /(d+3)} .
$$

Consequently, direction calculations, the generalized Hölder inequality, the Sobolev embedding, and the assumption (4.10) imply that

$$
\begin{aligned}
& \sum_{l=1}^{m-1} \int_{0}^{T} \int_{\Omega}\left|\nabla^{m+2} h_{N}\right|\left|\nabla^{m-1}\left[G\left(\left|\nabla h_{N}\right|^{2}\right) \nabla h_{N}\right]\right| d x d t \\
& \quad \leq C \int_{0}^{T} \int_{\Omega}\left(\left|\nabla^{5} h_{N}\right|\left|\nabla^{2} h_{N}\right|^{2}\left|\nabla h_{N}\right|+\left|\nabla^{5} h_{N}\right|\left|\nabla^{3} h_{N}\right|\left|\nabla h_{N}\right|^{2}\right) d x d t \\
& \quad \leq C \int_{0}^{T}\left\|h_{N}\right\|_{H^{5}(\Omega)}\left(\left\|h_{N}\right\|_{W^{2,6}(\Omega)}^{2}\|h\|_{W^{1,6}(\Omega)}+\|h\|_{W^{3,6}(\Omega)}\|h\|_{W^{1,6}(\Omega)}^{2}\right) d t \\
& \quad \leq C \int_{0}^{T}\left\|h_{N}\right\|_{H^{5}(\Omega)}\left\|h_{N}\right\|_{H^{4}(\Omega)}\left(\|h\|_{H^{2}(\Omega)}^{(d+9) /(d+3)}+\|h\|_{H^{2}(\Omega)}^{2}\right) d t \\
& \quad \leq C \int_{0}^{T}\left\|h_{N}\right\|_{H^{m+2}(\Omega)}\left\|h_{N}\right\|_{H^{m+1}(\Omega)} d t
\end{aligned}
$$

Now assume that $m \geq 4$. Notice from (4.10) and the Sobolev embedding $H^{2}(\Omega) \hookrightarrow$ $L^{\infty}(\Omega)$ that $\left|\nabla^{k} h_{N}(x)\right| \leq C$ if $k \leq m-3$. Thus, since the highest order of derivatives in $\partial^{l}\left[G\left(\left|\nabla h_{N}\right|^{2}\right)\right]$ is $l+1$, all $\left|\nabla^{l}\left[G\left(\left|\nabla h_{N}\right|^{2}\right)\right]\right| \leq C$ with $l \leq m-4$. Consequently,

$$
\begin{aligned}
& \sum_{l=1}^{m-1} \int_{0}^{T} \int_{\Omega}\left|\nabla^{m+2} h_{N}\right|\left|\nabla^{m-l} h_{N}\right|\left|\nabla^{l}\left[G\left(\left|\nabla h_{N}\right|^{2}\right)\right]\right| d x d t \\
& \leq C \int_{0}^{T}\left\|h_{N}\right\|_{H^{m+2}(\Omega)}\left\|h_{N}\right\|_{H^{m+1}(\Omega)} d t \\
& \quad+C \sum_{l=m-3}^{m-1} \int_{0}^{T} \int_{\Omega}\left|\nabla^{m+2} h_{N}\right|\left|\nabla^{m-l} h_{N}\right|\left|\nabla^{l}\left[G\left(\left|\nabla h_{N}\right|^{2}\right)\right]\right| d t
\end{aligned}
$$

which, together with (4.12), leads to

$$
\begin{aligned}
& \left\|h_{N}\right\|_{L^{\infty}\left(0, T ; H^{m}(\Omega)\right)}^{2}+\left\|h_{N}\right\|_{L^{2}\left(0, T ; H^{m+2}(\Omega)\right)}^{2} \\
& \quad \leq C \sum_{l=m-3}^{m-1} \int_{0}^{T} \int_{\Omega}\left|\nabla^{m+2} h_{N}\right|\left|\nabla^{m-l} h_{N}\right|\left|\nabla^{l}\left[G\left(\left|\nabla h_{N}\right|^{2}\right)\right]\right| d x d t
\end{aligned}
$$


Applying Lemma 3.4 and repeatedly using the fact that $\left|\nabla^{k} h_{N}\right| \leq C$ for $k \leq m-3$, we obtain by a series of calculations that

$$
\begin{aligned}
&\left|\nabla^{m-3}\left[G\left(\left|\nabla h_{N}\right|^{2}\right)\right]\right| \leq C\left|\nabla^{m-3}\left(\left|\nabla h_{N}\right|^{2}\right)\right| \leq C \sum_{j=2}^{m-2}\left|\nabla^{j} h_{N}\right|, \\
&\left|\nabla^{m-2}\left[G\left(\left|\nabla h_{N}\right|^{2}\right)\right]\right| \leq C\left|\nabla^{m-2}\left(\left|\nabla h_{N}\right|^{2}\right)\right|+C\left|\nabla^{m-3}\left(\left|\nabla h_{N}\right|^{2}\right)\right|\left|\nabla\left(\left|\nabla h_{N}\right|^{2}\right)\right| \\
& \leq C\left(\sum_{j=2}^{m-2}\left|\nabla^{j} h_{N}\right|\right)\left|\nabla^{2} h_{N}\right|+\sum_{j=3}^{m-1}\left|\nabla^{j} h_{N}\right|, \\
&\left|\nabla^{m-1}\left[G\left(\left|\nabla h_{N}\right|^{2}\right)\right]\right| \leq C\left|\nabla^{m-1}\left(\left|\nabla h_{N}\right|^{2}\right)\right|+C\left|\nabla^{m-2}\left(\left|\nabla h_{N}\right|^{2}\right)\right|\left|\nabla\left(\left|\nabla h_{N}\right|^{2}\right)\right| \\
& \quad+C\left|\nabla^{m-3}\left(\left|\nabla h_{N}\right|^{2}\right)\right|\left[\left|\nabla\left(\left|\nabla h_{N}\right|^{2}\right)\right|^{2}+\left|\nabla^{2}\left(\left|\nabla h_{N}\right|^{2}\right)\right|\right] \\
& \leq C \sum_{j=4}^{m}\left|\nabla^{j} h_{N}\right|+C\left(\sum_{j=3}^{m-1}\left|\nabla^{j} h_{N}\right|\right)\left|\nabla^{2} h_{N}\right| \\
& \quad+C\left(\sum_{j=2}^{m-2}\left|\nabla^{j} h_{N}\right|\right)\left(\left|\nabla^{2} h_{N}\right|^{2}+\left|\nabla^{3} h_{N}\right|\right) .
\end{aligned}
$$

It thus follows from (4.14), the generalized Hölder inequality, the Sobolev embedding, and (4.10) that

$$
\begin{aligned}
& \left\|h_{N}\right\|_{L^{\infty}\left(0, T ; H^{m}(\Omega)\right)}^{2}+\left\|h_{N}\right\|_{L^{2}\left(0, T ; H^{m+2}(\Omega)\right)}^{2} \\
& \leq C \int_{0}^{T}\left\|h_{N}\right\|_{H^{m+2}(\Omega)}\left(\left\|h_{N}\right\|_{W^{m-1,4}(\Omega)}\left\|h_{N}\right\|_{W^{m-2,4}(\Omega)}\right. \\
& \left.\quad \quad \quad\left\|h_{N}\right\|_{W^{m-2,6}(\Omega)}^{2}\left\|h_{N}\right\|_{W^{m-2,6}(\Omega)}\left\|h_{N}\right\|_{H^{m}(\Omega)}\right) d t \\
& \quad \leq C \int_{0}^{T}\left\|h_{N}\right\|_{H^{m+2}(\Omega)}\left(\left\|h_{N}\right\|_{H^{m}(\Omega)}\left\|h_{N}\right\|_{H^{m-1}(\Omega)}+\left\|h_{N}\right\|_{H^{m-1}(\Omega)}^{2}\left\|h_{N}\right\|_{W^{m-1,6}(\Omega)}\right) d t \\
& \leq C \int_{0}^{T}\left\|h_{N}\right\|_{H^{m+2}(\Omega)}\left\|h_{N}\right\|_{H^{m+1}(\Omega)} d t .
\end{aligned}
$$

This, together with (4.12), (4.13), Young's inequality, and (4.10), implies that

$$
\left\|h_{N}\right\|_{L^{\infty}\left(0, T ; H^{m}(\Omega)\right)}+\left\|h_{N}\right\|_{L^{2}\left(0, T ; H^{m+2}(\Omega)\right)} \leq C .
$$

Finally, setting $\phi=\left(\partial^{m-2}\right)^{2} \partial_{t} h_{N}(\cdot, t) \in H_{N}$ in (4.1) for each $t \in(0, T]$ and then integrating against $t$, we have by integration by parts and using (4.10), (4.15), and Lemma 3.2 that

$$
\left\|\partial_{t} h_{N}\right\|_{L^{2}\left(0, T ; H^{m-2}(\Omega)\right)}^{2} \leq C+C \int_{0}^{T} \int_{\Omega}\left|\nabla^{m-2} \partial_{t} h_{N}\right|\left|\nabla^{m-1}\left[G\left(\left|\nabla h_{N}\right|^{2}\right) \nabla h_{N}\right]\right| d x d t
$$

Comparing this with (4.11), we obtain by the same argument used above that

$$
\left\|\partial_{t} h\right\|_{L^{2}\left(0, T ; H^{m-2}(\Omega)\right)} \leq C .
$$

which, together with (4.15) imply (4.3) for $m \geq 3$. 
Proof of Theorem 4.3. Fix $N \geq 1$. A direct calculation using Fourier expansion implies that

$$
\left\|h-\mathcal{P}_{N} h\right\|_{L^{2}(\Omega)} \leq C N^{-m}\|h\|_{H^{m}(\Omega)} \quad \text { a.e. } t \in(0, T) .
$$

By the definition of $\mathcal{P}_{N}: L^{2}(\Omega) \rightarrow H_{N}$ and (3.4), we have for any $\phi \in H_{N}$ that

$$
\begin{gathered}
\left\langle\phi, \partial_{t} \mathcal{P}_{N} h\right\rangle+\left\langle\nabla \phi, G\left(\left|\nabla \mathcal{P}_{N} h\right|^{2}\right) \nabla \mathcal{P}_{N} h\right\rangle-\left\langle\nabla \phi, \nabla \mathcal{P}_{N} h\right\rangle+\delta\left\langle\Delta \phi, \Delta \mathcal{P}_{N} h\right\rangle \\
=\left\langle\nabla \phi, G\left(\left|\nabla \mathcal{P}_{N} h\right|^{2}\right) \nabla \mathcal{P}_{N} h-G\left(|\nabla h|^{2}\right) \nabla h\right\rangle \quad \text { a.e. } t \in(0, T) .
\end{gathered}
$$

With $w_{N}=\mathcal{P}_{N} h(\cdot, t)-h_{N}(\cdot, t) \in H_{N}$ for $t \in(0, T)$, this, together with (4.1), leads to

$$
\begin{gathered}
\left\langle\phi, \partial_{t} w_{N}\right\rangle+\left\langle\nabla \phi, G\left(\left|\nabla \mathcal{P}_{N} h\right|^{2}\right) \nabla \mathcal{P}_{N} h-G\left(\left|\nabla h_{N}\right|^{2}\right) \nabla h_{N}\right\rangle-\left\langle\nabla \phi, \nabla w_{N}\right\rangle+\delta\left\langle\Delta \phi, \Delta w_{N}\right\rangle \\
=\left\langle\nabla \phi, G\left(\left|\nabla \mathcal{P}_{N} h\right|^{2}\right) \nabla \mathcal{P}_{N} h-G\left(|\nabla h|^{2}\right) \nabla h\right\rangle \quad \text { a.e. } t \in(0, T) .
\end{gathered}
$$

Setting $\phi=w_{N} \in H_{N}$, applying Lemma 3.3 with $a=\nabla \mathcal{P}_{N} h$ and $b=\nabla h_{N}$, the CauchySchwarz inequality, the Sobolev embedding $H^{4}(\Omega) \hookrightarrow W^{2, \infty}(\Omega)$, and Lemma 3.2, we have

$$
\begin{aligned}
\frac{1}{2} \frac{d}{d t}\left\|w_{N}\right\|^{2}-\left\|\nabla w_{N}\right\|^{2}+\delta\left\|\Delta w_{N}\right\|^{2} \\
\quad \leq\left|\left\langle\nabla w_{N},\left[G\left(\left|\nabla \mathcal{P}_{N} h\right|^{2}\right)-G\left(|\nabla h|^{2}\right)\right] \nabla \mathcal{P}_{N} h\right\rangle\right|+\left|\left\langle\nabla w_{N}, G\left(|\nabla h|^{2}\right) \nabla\left(\mathcal{P}_{N} h-h\right)\right\rangle\right| \\
\quad=\left|\left\langle\left(\nabla w_{N} \cdot \nabla \mathcal{P}_{N} h\right), G\left(\left|\nabla \mathcal{P}_{N} h\right|^{2}\right)-G\left(|\nabla h|^{2}\right)\right\rangle\right|+\left|G\left(|\nabla h|^{2}\right) \nabla w_{N}, \nabla\left(\mathcal{P}_{N} h-h\right)\right\rangle \mid \\
\quad=\left|\left\langle\nabla \cdot\left[\left(\nabla w_{N} \cdot \nabla \mathcal{P}_{N} h\right) Q_{N}\right], \mathcal{P}_{N} h-h\right\rangle\right|+\left|\nabla \cdot\left[G\left(|\nabla h|^{2}\right) \nabla w_{N}\right], \mathcal{P}_{N} h-h\right\rangle \mid,
\end{aligned}
$$

where

$$
Q_{N}= \begin{cases}\frac{\nabla \mathcal{P}_{N} h+\nabla h}{\left(1+\left|\nabla \mathcal{P}_{N}\right|^{2}\right)\left(1+|\nabla h|^{2}\right)} & \text { if } G=G_{1} \\ \nabla \mathcal{P}_{N}+\nabla h & \text { if } G=G_{2}\end{cases}
$$

Therefore,

$$
\begin{aligned}
\frac{1}{2} \frac{d}{d t}\left\|w_{N}\right\|^{2}-\left\|\nabla w_{N}\right\|^{2}+\delta\left\|\Delta w_{N}\right\|^{2} \\
\quad \leq C\left(\|h\|_{W^{2, \infty}(\Omega)}^{2}+\left\|\mathcal{P}_{N} h\right\|_{W^{2, \infty}(\Omega)}^{2}\right)\left\|\mathcal{P}_{N} h-h\right\|\left(\left\|\nabla w_{N}\right\|+\left\|\Delta w_{N}\right\|\right) \\
\quad \leq C\left\|\mathcal{P}_{N} h-h\right\|\left\|\nabla w_{N}\right\|+C\left\|\mathcal{P}_{N} h-h\right\|\left\|\Delta w_{N}\right\| \\
\quad \leq C\left\|\mathcal{P}_{N} h-h\right\|^{2}+C\left\|\nabla w_{N}\right\|^{2}+\frac{\delta}{2}\left\|\Delta w_{N}\right\|^{2} \quad \text { a.e. } t \in(0, T) .
\end{aligned}
$$

This, together with Lemma 3.1 and Young's inequality, implies that

$$
\begin{aligned}
\frac{d}{d t}\left\|w_{N}\right\|^{2} & \leq C\left\|\mathcal{P}_{N} h-h\right\|^{2}+C\left\|\nabla w_{N}\right\|^{2}-\delta\left\|\Delta w_{N}\right\|^{2} \\
& \leq C\left\|\mathcal{P}_{N} h-h\right\|^{2}+C\left\|w_{N}\right\|^{2}-\frac{\delta}{2}\left\|\Delta w_{N}\right\|^{2},
\end{aligned}
$$

The desired error estimate now follows from the Gronwall inequality, the fact that $w_{N}(0)=0$, and the estimate (4.16). 


\section{Numerical Simulation}

To simulate the growth dynamics numerically, we have developed the following spectral method for the initial-boundary-value problem (3.1)-(3.3).

Step 1: Splitting. Rewrite the underlying equation as

$$
\left(\partial_{t}+\frac{\delta}{2} \Delta^{2}+\Delta\right) h=A(\nabla h)-\frac{\delta}{2} \Delta^{2} h,
$$

where $A(\nabla h)=\nabla \cdot\left[G\left(|\nabla h|^{2}\right) \nabla h\right]$.

Step 2: Spectral approximation. Perform the Fourier transform to get

$$
\left(\frac{d}{d t}+\frac{\delta}{2}|k|^{4}-|k|^{2}\right) \hat{h}_{k}(t)=\hat{A}_{k}(t)-\frac{\delta}{2}|k|^{4} \hat{h}_{k}(t) \quad \forall k:|k| \leq \frac{N}{2},
$$

where $\hat{h}_{k}(t)$ and $\hat{A}_{k}(t)$ with $|k| \leq N / 2$ are the Fourier modes at time $t$ of the solution $h(\cdot, t)$ and the nonlinear term $A(\nabla h(\cdot, t))$, respectively, and $N \geq 1$ is a fixed integer.

Step 3: Reformulation with integrating factor. Rearrange the ordinary differential equation with an integration factor to get

$$
e^{-\left(\frac{\delta}{2}|k|^{4}-|k|^{2}\right) t} \frac{d}{d t}\left[e^{\left(\frac{\delta}{2}|k|^{4}-|k|^{2}\right) t} \hat{h}_{k}(t)\right]=\hat{A}_{k}(t)-\frac{\delta}{2}|k|^{4} \hat{h}_{k}(t) \quad \forall k:|k| \leq \frac{N}{2} .
$$

Step 4: Crank-Nicolson and Adam-Bashforth discretization. Use the Crank-Nicolson and Adam-Bashforth schemes to discretize the surface diffusion and nonlinear terms, respectively, to get

$$
\begin{gathered}
e^{-\left(\frac{\delta}{2}|k|^{4}-|k|^{2}\right) t_{n+1 / 2}} \frac{\left[e^{\left(\frac{\delta}{2}|k|^{4}-|k|^{2}\right) t_{n+1}} \hat{h}_{k}^{n+1}(t)-e^{\left(\frac{\delta}{2}|k|^{4}-|k|^{2}\right) t_{n}} \hat{h}_{k}^{n}(t)\right]}{\Delta t} \\
=\frac{3 \hat{A}_{k}^{n}-\hat{A}_{k}^{n-1}}{2}-\frac{\delta}{2}|k|^{4} \frac{\hat{h}_{k}^{n}+\hat{h}_{k}^{n+1}}{2} \quad \forall k:|k| \leq \frac{N}{2},
\end{gathered}
$$

where $\hat{h}_{k}^{n}$ and $\hat{A}_{k}^{n}$ approximate $\hat{h}_{k}\left(t_{n}\right)$ and $\hat{A}_{k}\left(t_{n}\right)$, respectively, $t_{n}$ is the $n$-th time step, and $\Delta t$ is the time stepping. This can be simplified to

$$
\begin{aligned}
& \frac{e^{\left(\frac{\delta}{2}|k|^{4}-|k|^{2}\right) \frac{\Delta t}{2}} \hat{h}_{k}^{n+1}-e^{-\left(\frac{\delta}{2}|k|^{4}-|k|^{2}\right) \frac{\Delta t}{2}} \hat{h}_{k}^{n}}{\Delta t} \\
&=\frac{3 \hat{A}_{k}^{n}-\hat{A}_{k}^{n-1}}{2}-\frac{\delta|k|^{4}}{4}\left(\hat{h}_{k}^{n}+\hat{h}_{k}^{n+1}\right) \quad \forall k:|k| \leq \frac{N}{2} .
\end{aligned}
$$

Step 5. Solve the system and perform the inverse Fourier transform. This will give the numerical solution at different time steps.

Using this numerical method, we solve the initial-boundary-value problem (3.1)-(3.3) with the parameter $\delta=0.1$, the space dimension $d=2$, the domain $\Omega=(0,2 \pi) \times(0,2 \pi)$, and the initial data

$$
h_{0}(x)=0.1\left(\sin 3 x_{1} \sin 2 x_{2}+\sin 5 x_{1} \sin 5 x_{2}\right) \quad x \in \Omega .
$$

In this case, the critical modulus of wavevector is $k_{c}=\sqrt{10}$, cf. (2.10), and the most unstable modes are those with wavevectors $k$ such that $|k|=\sqrt{5}$. The initial data $h_{0}$ consists of only two linearly stable modes $k_{1}=(3,2)$ and $k_{2}=(5,5)$. However, their interaction generates an unstable mode $k_{2}-2 k_{1}=(-1,1)$, cf. (2.14). 
For a height profile $h=h(x, t)$, we denote its energy by $E(t)=E(h(t))$, where $E(h)=$ $E_{1}(h)$ in the case without slope selection and $E(h)=E_{2}(h)$ in the case with slope selection, cf. (1.3) and (1.4). We also define its interface width or roughness by

$$
w(t)=\sqrt{\frac{1}{|\Omega|} \int_{\Omega}[h(x, t)-\bar{h}(t)]^{2} d x}, \quad \text { where } \bar{h}(t)=\frac{1}{|\Omega|} \int_{\Omega} h(x, t) d x .
$$

In our contour plots below, we use the coordinate $(x, y)$ instead of $\left(x_{1}, x_{2}\right)$.
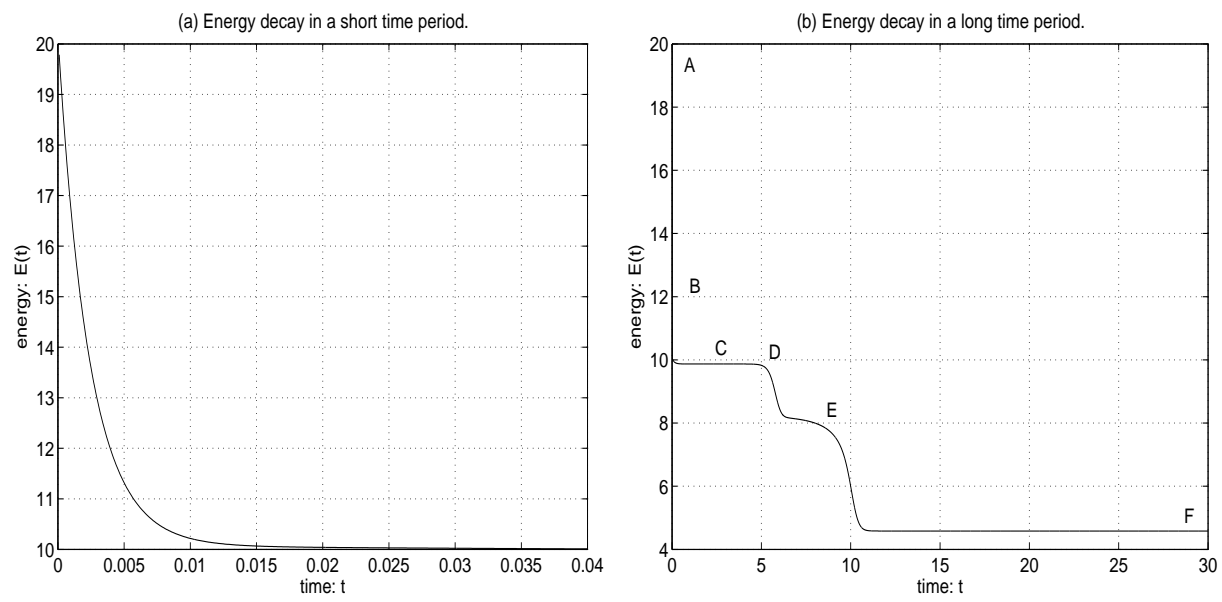

Figure 5.1. The decay of energy for the growth with slope selection.
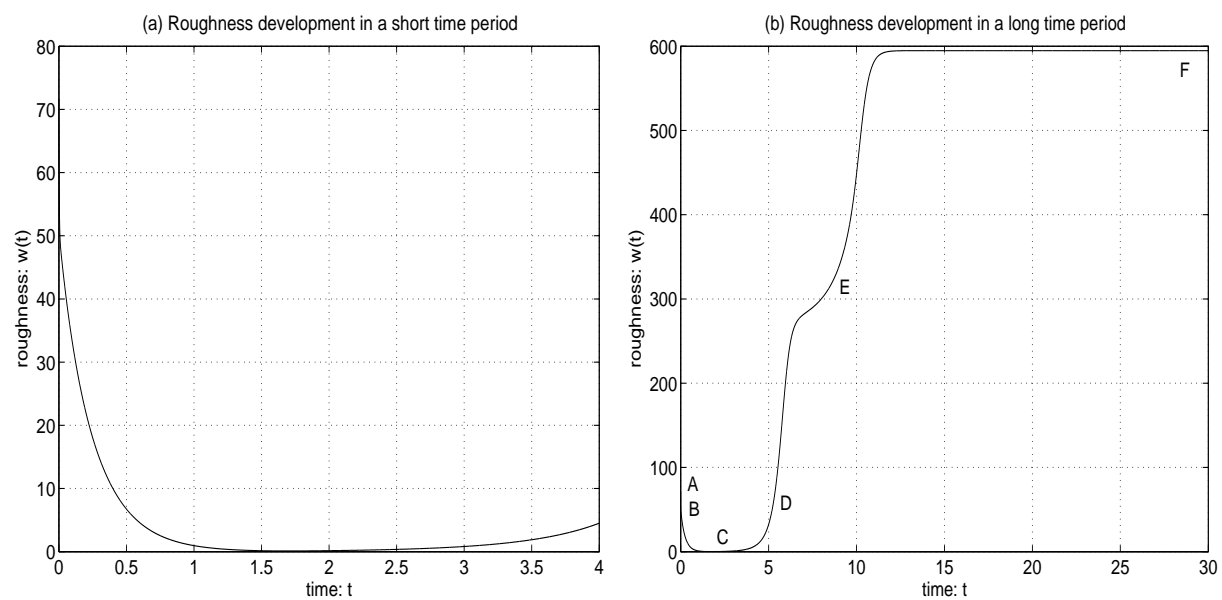

Figure 5.2. The development of roughness for the growth with slope selection.

1. The growth dynamics with slope selection. Figure 5.1 and Figure 5.2 show the decay of energy $E(t)$ and the development of profile roughness $w(t)$, respectively. We observe that initially both the energy and roughness decay rapidly. However, after a relatively long period of time, the roughness starts to grow. This is exactly the morphological instability in the rough-smooth-rough pattern that is analyzed in Section 2. Notice that the flat tail in Figure 5.1 (b) and Figure 5.2 (b) indicate that a steady-state solution is reached. The letters A, B, C, D, E, and F marked on Figure 5.1 (b) and Figure 5.2 (b) indicate different 
coarsening stages at time $t=0,0.05,2.5,5.5,8$, and 30 , respectively. There is a structural transition from each stage to the immediate next one.
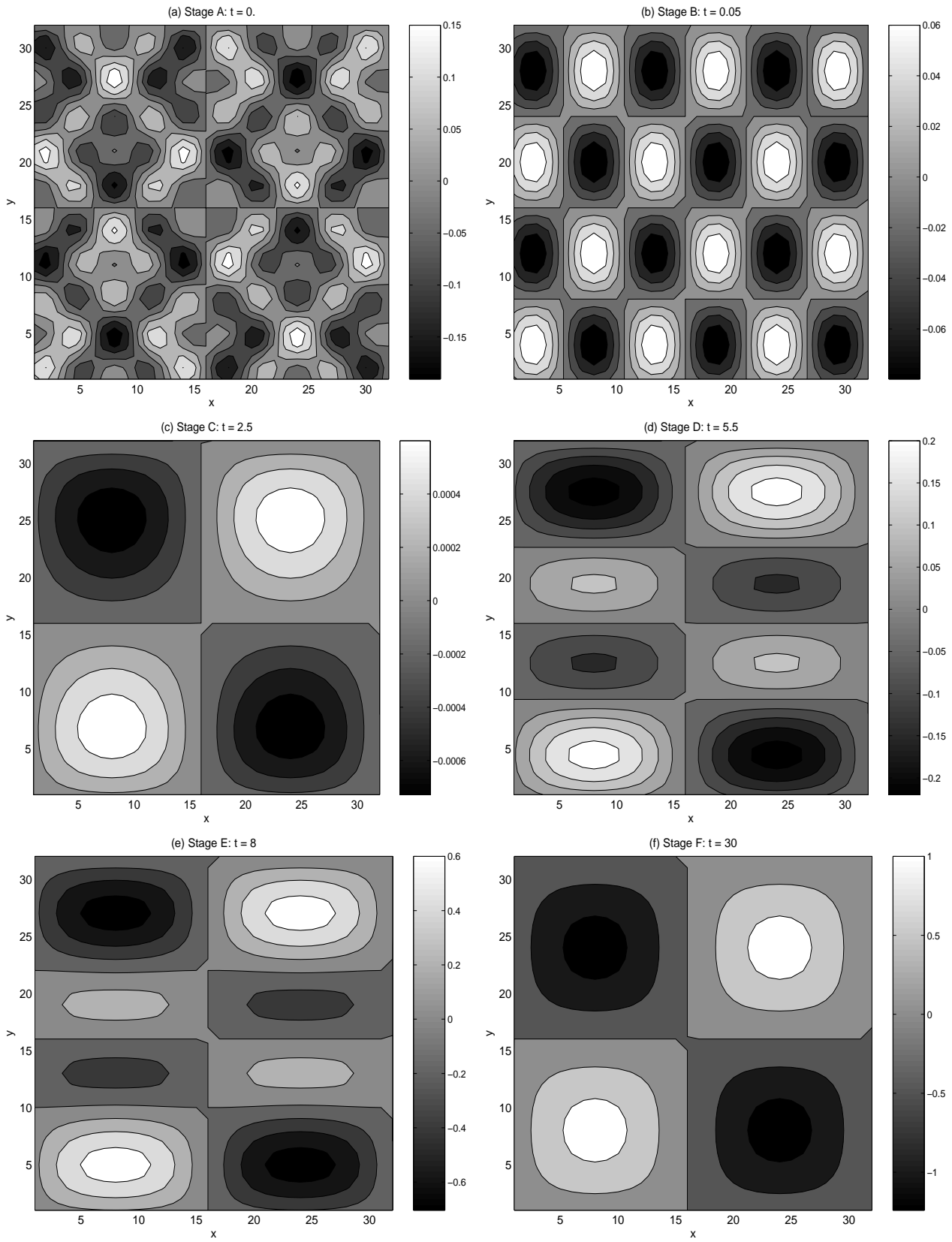

Figure 5.3. Contour plots of height profiles for the growth with slope selection.

Figure 5.3 shows contour plots in grey scale of the height profile $h$ corresponding to the four stages A, B, C, D, E, and F as marked in Figure 5.1 (b) and Figure 5.2 (b). At Stage $\mathrm{B}$, the initial mode $(5,5)$ has disappeared but $(3,2)$ still exists. Note that mound or pyramid base boundaries are straightened locally. At Stage $\mathrm{C}$, both of these initially linearly stable 
modes are gone. But the mode $(-1,1)$ which is generated from these modes appears. Observe that at Stage D a new mode $(1,2)$ appears due to further interaction of different modes in the profile. Notice that $(1,2)$ is one of the most unstable modes. But this mode almost disappears at Stage E. Finally, Figure 5.3 (f) shows a steady-state profile which consists mainly of only one mode $(1,1)$. Comparing Figure 5.3 with Figure 5.1 and Figure 5.2, we observe that, after the initial preparation, the coarsening dynamics is characterized by a fast structural transition and a very slow motion in between such transitions.

Figure 5.4 shows contour plots of the profile gradient $|\nabla h|$ at those marked stages. 

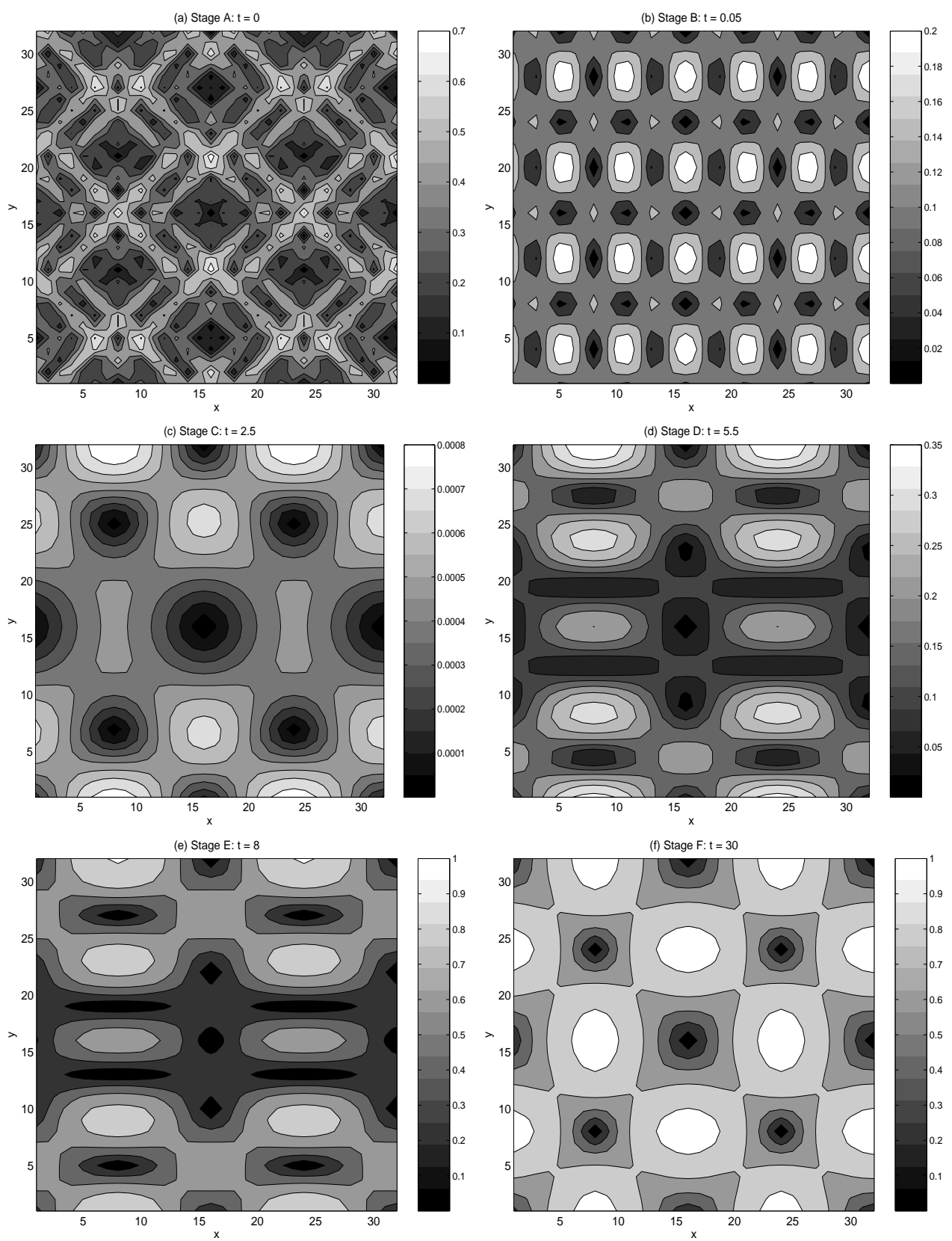

Figure 5.4. Contour plots of gradients for the growth with slope selection.

2. The growth dynamics without slope selection. Figure 5.5 and Figure 5.6 show the decay of energy $E(t)$ and the development of profile roughness $w(t)$, respectively. They are similar to those for the growth dynamics with slope selection. In particular, the initial rough-smoothrough pattern is the same. But, in this case, the energy becomes negative. The letters A, B, C, D, E, and F marked on Figure 5.5 (b) and Figure 5.6 (b) correspond to different coarsening stages at time $t=0,0.05,2.5,5.5,8$, and 30, respectively. For the purpose of comparison, we have chosen them to be the same as for the case with slope selection. 

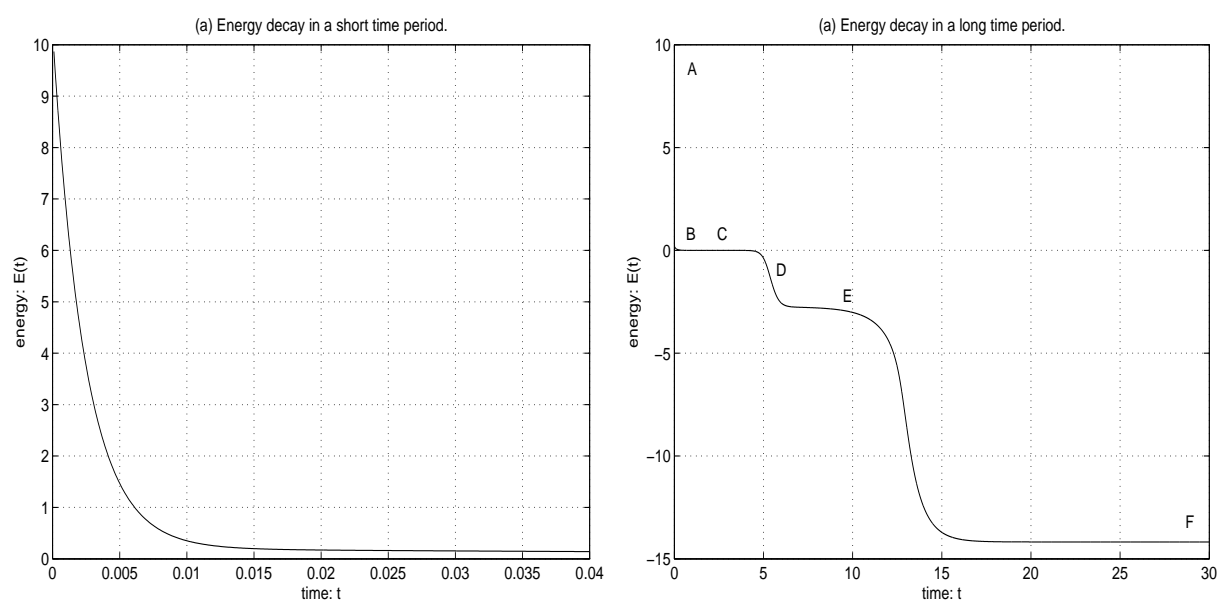

Figure 5.5. The decay of energy for the growth without slope selection.
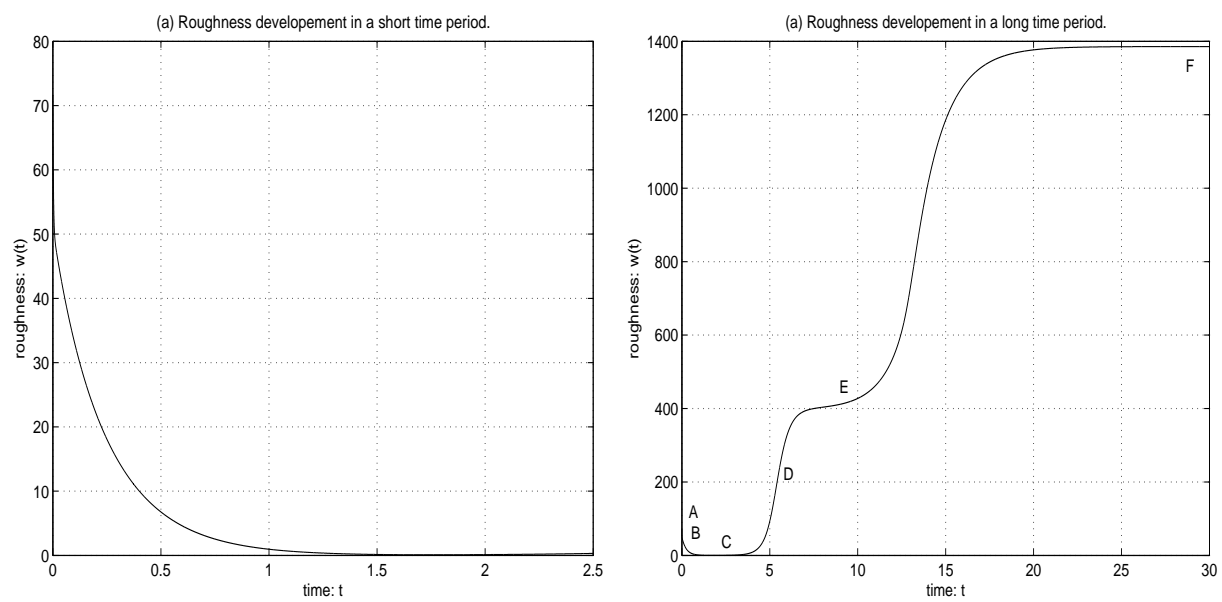

Figure 5.6. The development of roughness for the growth without slope selection.

Figure 5.7 and Figure 5.8 show contour plots of the height profile $h$ and the magnitude of its gradient $|\nabla h|$ at the different stages marked on Figure 5.5 (b) and Figure 5.6 (b). With regards to structural transition in the coarsening, they are similar to those plots in Figure 5.3 and Figure 5.4 for the growth dynamics with slope selection. But, from the corresponding grey scale bars, we see clearly that the profile gradient for the growth without slop selection is much larger in magnitude than that for the growth with slope selection.

\section{Conclusions}

We have rigorously proved that the initial-boundary-value problem of the growth equation (1.1) or (1.2) is well-posed. Both of our weakly nonlinear analysis and numerical simulation reveal a nonlinear morphological instability in the rough-smooth-rough pattern at an early stage of growth, agreeing with reported experiments on thin film epitaxy on rough surfaces. In this early stage, linearly stable modes are damped immediately. But their nonlinear interaction possibly generates new unstable modes. Mound or pyramid base boundaries are immediately straightened locally. This is different from phase boundaries in the Cahn-Hilliard 

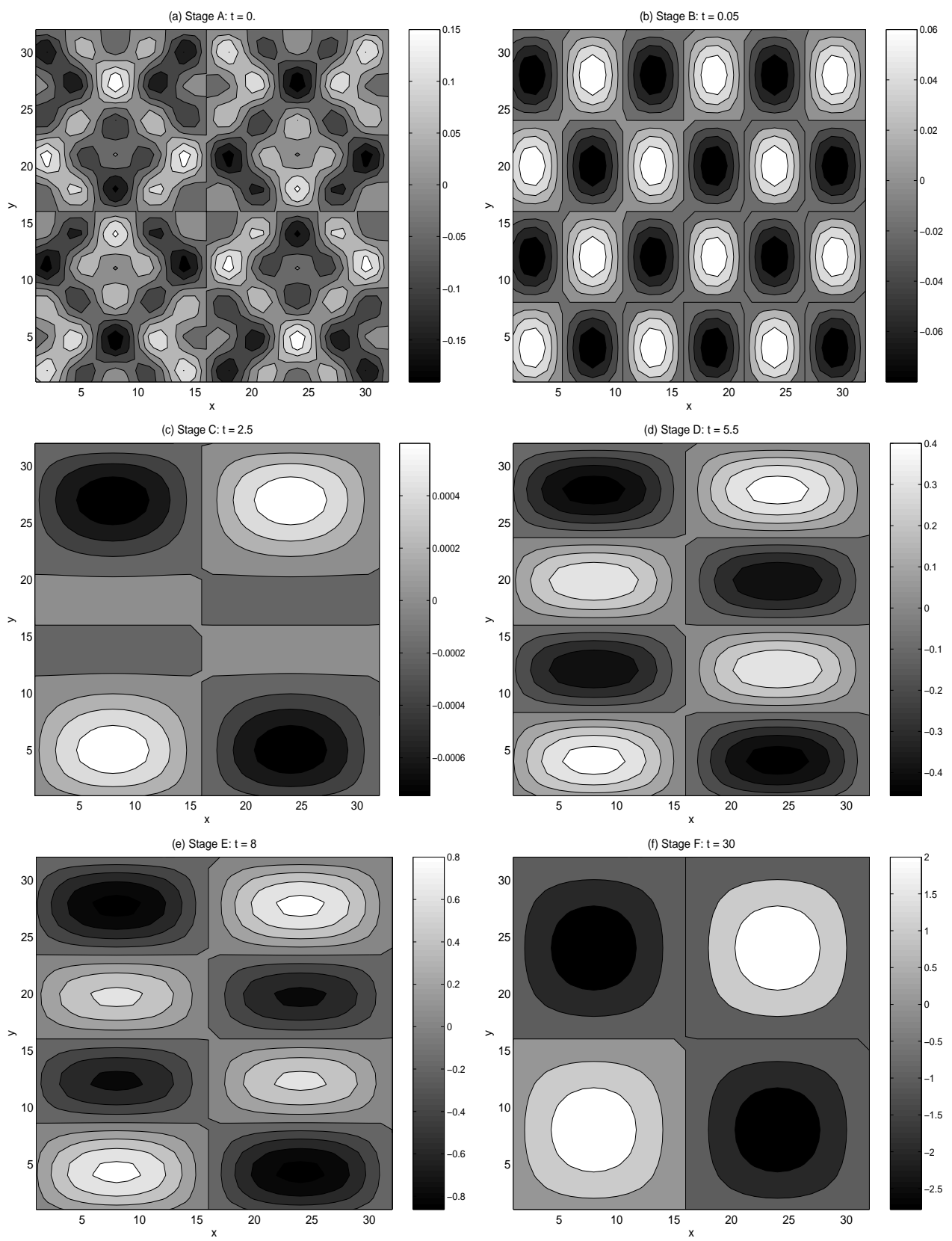

Figure 5.7. Contour plots of height profiles for the growth without slope selection.

coarsening. We have also developed a spectral numerical method for simulating the coarsening dynamics governed by the underlying equations, with or without slope selection. Our numerical results clearly distinguish different stages of coarsening such as the initial preparation and the alternating rapid structural transition and slow motion. They also display a large mound slope that characterizes the coarsening dynamics without slope selection. More simulations with various kinds of initial data with or without noise are required to predict the exact roughness component for both of the growth laws. 

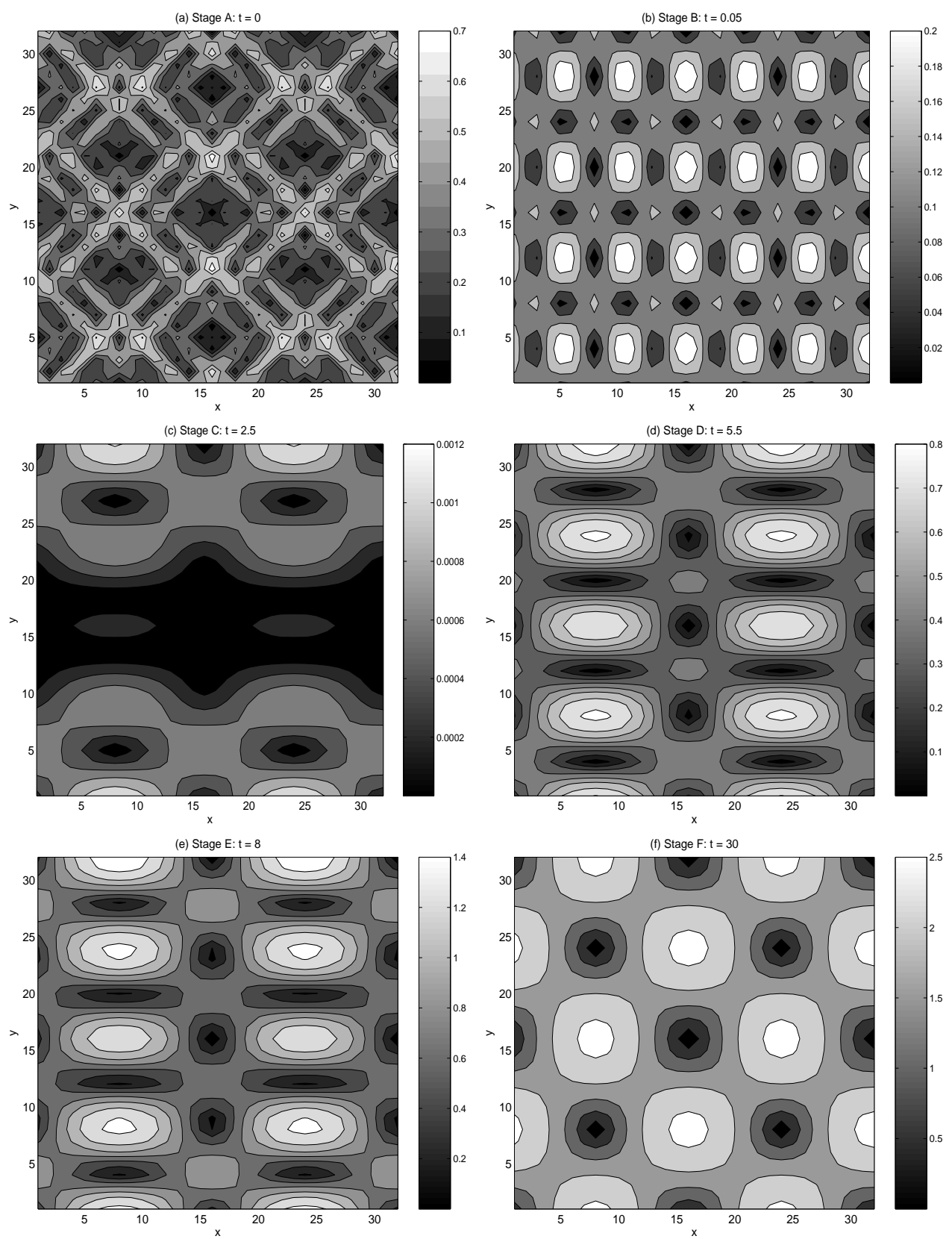

Figure 5.8. Contour plots of gradients for the growth without slope selection.

Acknowledgment. This work was partially supported by the National Science Foundation of the United States through grant DMS-0072958 and DMS-0107218. The authors thank Dr. Robert Kohn, Dr. Christian Ratsch, and Dr. Oliver Stein for bringing to their attention the references [20], [11], and [18], respectively.

\section{REFERENCES}

[1] P. Aviles and Y. Giga. A mathematical problem related to the physical theory of liquid crystal configurations. Proc. Centre Math. Anal. Austral. Nat. Univ., 12:1-16, 1987. 
[2] G. S. Bales and A. Zangwill. Morphological instability of a terrace edge during step-flow growth. Phys. Rev. B, 41(9):5500-5508, 1990.

[3] J. M. Ball and R. D. James. Proposed experimental tests of a theory of fine microstructure and the two-well problem. Phil. Trans. R. Soc. Lond. A, 338:389-450, 1992.

[4] A.-L. Barabási and H. E. Stanley. Fractal Concepts in Surface Growth. Cambridge University Press, 1995.

[5] J. W. Cahn and J. E. Hilliard. Free energy of a nonuniform system I. Interfacial free energy. J. Chem. Phys., 28:258-267, 1958.

[6] B. D. Coleman, R. S. Falk, and M. Moakher. Stability of cylindrical bodies in the theory of surface diffusion. Physica D, 89, 1995.

[7] G. Ehrlich and F. G. Hudda. Atomic view of surface diffusion: tungsten on tungsten. J. Chem. Phys., 44:1036, 1966.

[8] L. C. Evans. Partial Differential Equations, volume 19 of Graduate Studies in Mathematics. AMS, 1998.

[9] G. Gioia and M. Ortiz. Delamination of compressed thin films. Adv. Appl. Mech., 33:119-192, 1997.

[10] L. Golubović. Interfacial coarsening in epitaxial growth models without slope selection. Phys. Rev. Lett., 78(1):90-93, 1997.

[11] M. F. Gyure, J. J. Zinck, C. Ratsch, and D. D. Vvedensky. Unstable growth on rough surface. Phys. Rev. Lett, 81(22):4931-4934, 1998.

[12] C. Herring. Surface tension as a motivation for sintering. In W. E. Kingston, editor, The Physics of Powder Metallurgy, pages 143-179. McGraw-Hill, 1951.

[13] C. Herring. The use of classical macroscopic concepts in surface energy problems. In R. Gomer and C. S. Smith, editors, Structure and Properties of Solid Surfaces, pages 2-72. Univ. of Chicago Press, 1953.

[14] W. Jin and R. V. Kohn. Singular perturbation and the energy of folds. J. Nonlinear Sci., 10:355-390, 2000.

[15] M. D. Johnson, C. Orme, A. W. Hunt, D. Graff, J. Sudijono, L. M. Sander, and B. G. Orr. Stable and unstable growth in molecular beam epitaxy. Phys. Rev. Lett., 72(1):116-1119, 1994.

[16] W. Jost. Diffusion in solids, liquids, gases. Academic Press, 1952.

[17] S. V. Khare and T. L. Einstein. Unified view of step-edge kinetics and fluctuations. Phys. Rev. B, 57(8):4782-4797, 1998.

[18] B. B. King, O. Stein, and M. Winkler. A fourth order parabolic equation modelling epitaxial thin film growth. Preprint No. 94, Department of Mathematics - C, Aachen University, 2000.

[19] R. Kohn and S. Müller. Surface energy and microstructure in coherent phase transitions. Comm. Pure Appl. Math., 47:405-435, 1994.

[20] R. V. Kohn and X. Yan. Upper bounds on the coarsening rate for an epitaxial growth model. Comm. Pure Appl. Math., 2002 (submitted).

[21] J. Krug. Surface diffusion currents and the universality classes of growth. Phys. Rev. Lett., 70(21):3271$3274,1993$.

[22] V. G. Maz'ja. Sobolev Spaces. Springer-Verlag, 1985.

[23] D. Moldovan and L. Golubović. Interfacial coarsening dynamics in epitaxial growth with slope selection. Phys. Rev. E, 61(6):6190-6214, 2000.

[24] W. W. Mullins. Theory of thermal grooving. J. Appl. Phys., 28:333-339, 1957.

[25] M. Ortiz, E. Repetto, and H. Si. A continuum model of kinetic roughening and coarsening in thin films. J. Mech. Phys. Solids, 47:697-730, 1999.

[26] A. Pimpinelli and J. Villain. Physics of Crystal Growth. Cambridge University Press, 1998.

[27] A. Pimpinelli, J. Villain, D. E. Wolf, J. J. Métois, J. C. Heyraud, I. Elkinani, and G. Uimin. Equilibrium step dynamics on vicinal surfaces. Surface Sci., 295:143, 1993.

[28] P. Politi, G. Grenet, A. Marty, A. Ponchet, and J. Villain. Instabilities in crystal growth by atomic or molecular beams. Phys. Reports, 324:271-404, 2000.

[29] P. Politi and J. Villain. Ehrlich-Schwoebel instability in molecular-beam epitaxy: A minimal model. Phys. Rev. B, 54(7):5114-5129, 1996.

[30] R. L. Schwoebel. Step motion on crystal surfaces II. J. Appl. Phys., 40:614, 1969. 
[31] R. L. Schwoebel and E. J. Shipsey. Step motion on crystal surfaces. J. Appl. Phys., 37:3682, 1966.

[32] M. Siegert and M. Plischke. Slope selection and coarsening in molecular beam epitaxy. Phys. Rev. Lett., 73(11):1517-1520, 1994.

[33] J. Simon. Compact sets in the space $L^{p}(0, T ; B)$. Ann. Math. Pure Appl., 146:65-96, 1987.

[34] R. Temam. Navier-Stokes Equations. North-Holland, 1984.

[35] J. Villain. Continuum models of crystal growth from atomic beams with and without desorption. J. de Phys. I, 1:19-42, 1991.

Department of Mathematics, University of Maryland, College Park, MD 20742-4015, USA.

E-mail address: bli@math.umd.edu.

Institute for Physical Science and Technology and Department of Mathematics, UniverSity of Maryland, College Park, MD 20742-4015, USA.

E-mail address: jliu@math.umd.edu. 